\title{
Boundary conditions for the Einstein evolution system
}

\author{
Lawrence E. Kidder, ${ }^{1}$ Lee Lindblom, ${ }^{2}$ Mark A. Scheel, ${ }^{2}$ Luisa T. Buchman, ${ }^{3}$ and Harald P. Pfeiffer ${ }^{2}$ \\ ${ }^{1}$ Center for Radiophysics and Space Research, Cornell University, Ithaca, New York 14853, USA \\ ${ }^{2}$ Theoretical Astrophysics 130-33, California Institute of Technology, Pasadena, California 91125, USA \\ ${ }^{3}$ Jet Propulsion Laboratory, California Institute of Technology, Pasadena, California 91109, USA
}

(Received 22 December 2004; published 18 March 2005)

\begin{abstract}
New boundary conditions are constructed and tested numerically for a general first-order form of the Einstein evolution system. These conditions prevent constraint violations from entering the computational domain through timelike boundaries, allow the simulation of isolated systems by preventing physical gravitational waves from entering the computational domain, and are designed to be compatible with the fixed-gauge evolutions used here. These new boundary conditions are shown to be effective in limiting the growth of constraints in 3D nonlinear numerical evolutions of dynamical black-hole spacetimes.
\end{abstract}

DOI: 10.1103/PhysRevD.71.064020

PACS numbers: 04.25.Dm, 02.60.Cb, 04.20.Cv

\section{INTRODUCTION}

The Einstein system can be written as a set of evolution equations that determine how the dynamical fields change with time, plus constraint equations that must be satisfied by the physically relevant field configurations. The evolution equations ensure that the constraints will be satisfied within the domain of dependence of the initial data if they are satisfied initially. But this does not guarantee that constraints that are initially small (rather than precisely zero) will remain small, or that constraint violations will not enter a domain through its timelike boundaries. Indeed, the rapid growth of constraint violations from small (truncation or even roundoff-level) values in the initial data continues to be one of the major problems for the numerical relativity community.

Constraint violations in the continuum evolution equations (as distinct from their discrete numerical approximations) have at least two different causes: (i) bulk constraint violations, in which existing violations are amplified by the evolution equations, and (ii) boundary violations, in which constraint violations flow into the domain through timelike boundaries. A variety of techniques have been introduced to control bulk constraint violations in numerical solutions of constrained evolution systems; these include constraint projection [1-3] (where the constraint equations are resolved whenever the constraints become too large), fully constrained evolution [4-11] (where some dynamical fields are determined at each time step by solving the constraint equations rather than their evolution equations), and dynamical constraint control [12-14] (in which the evolution equations are modified dynamically in a way that minimizes the growth of the constraints). Techniques have also been proposed to control boundary constraint violations; these include the construction of special forms of the evolution equations that prevent constraint violations from flowing into the domain $[15,16]$, and special boundary conditions that prevent the influx of constraint violations in more general forms of the evolution equations [3,14,1727].
Recent studies have shown that bulk constraint violations are not effectively controlled by constraint projection [3] or dynamical constraint control [14] techniques, unless the influx of boundary constraint violations is also controlled separately. Thus the explicit control of boundary constraint violations appears to be an essential requirement for effective constraint control in the Einstein system. The primary purpose of this paper is to construct and then test numerically the special boundary conditions needed to prevent the influx of constraint violations in one (rather general) first-order form of the Einstein evolution system: the multiparameter generalization of the Frittelli-Reula system [28] introduced by Kidder, Scheel, and Teukolsky (KST) [29]. These constraint-preserving boundary conditions are constructed here using the strategy first outlined by Stewart [17] for the Einstein equations. The idea is to decompose the constraint fields into incoming and outgoing parts, based on the characteristic decomposition of the constraint evolution equations. The incoming constraint fields are controlled (e.g. set to zero) at each boundary point, and these conditions then serve as boundary conditions for the principal dynamical fields of the system. The constraint-preserving boundary conditions derived here generalize earlier work by being applicable to generic nonlinear field configurations $[19,22]$, having no symmetry requirements [18], and allowing arbitrary values of the gauge fields (i.e., the lapse and shift) $[15,21]$. These new boundary conditions are also tested here under more challenging conditions - nonlinear 3D evolutions of dynamical black-hole spacetimes - than previously considered.

A secondary purpose of this paper is to introduce and test boundary conditions for the physical (gravitationalwave) degrees of freedom of the Einstein system. These new physical boundary conditions control the influx of the radiative part of the Weyl tensor, and generalize (to the generic $3 \mathrm{D}$ case) the boundary conditions of this type proposed by Bardeen and Buchman [18]. Boundary conditions are also introduced and tested here for those dynamical fields that are not fixed by the physical or the 
constraint-preserving boundary conditions. The boundary conditions for these extra "gauge" degrees of freedom are set in a way that is consistent with the "fixed-gauge" (i.e., time independent lapse density and shift) evolutions used in the numerical tests described here.

The remainder of this paper is organized as follows. The basic KST form of the Einstein evolution system is reviewed in Sec. II, and additional technical details (which considerably generalize previously published work) on the characteristic decomposition of the dynamical fields of this system are given in Appendix A. The constraint evolution equations associated with the KST system are described in Sec. III, and additional details on the hyperbolicity of the constraint system are given in Appendix B. The principal new analytical results of this paper are contained in Sec. IV, where constraint-preserving boundary conditions are derived for the KST system. Two types of constraintpreserving boundary conditions are presented here: the first, in Sec. IVA, are based on the characteristic constraint decomposition ideas of Stewart [17], while the second, in Sec. IV B, are generalizations of a simpler (but less generally applicable) type of constraint-preserving boundary condition which has been used effectively for the scalarwave system [3]. Physical boundary conditions that control the influx of the radiative part of the Weyl tensor are presented in Sec. V. Boundary conditions for the remaining gauge dynamical fields are given in Sec. VI. We test the efficacy of these new boundary conditions by performing numerical evolutions of various perturbed and unperturbed black-hole spacetimes. The basic numerical methods used in these tests are described in Sec. VII. The tests themselves are described in Sec. VIII: tests performed on unperturbed black holes are given in Sec. VIII A, tests on perturbed black holes are presented in Sec. VIII B, and finally a mild "angular" numerical instability that appears in some of these tests is discussed in Sec. VIII C. The major results of this paper are summarized and outstanding questions raised by this work are discussed in Sec. IX.

\section{PRINCIPAL EVOLUTION SYSTEM}

The form of the Einstein evolution system used here is a first-order formulation introduced by Kidder, Scheel, and Teukolsky [29], which generalizes several earlier forms of the Einstein system [28,30,31]. This system consists of first-order evolution equations for the spatial metric $g_{i j}$, the extrinsic curvature $K_{i j}$, and the spatial derivatives of the metric $D_{k i j}=\partial_{k} g_{i j} / 2$. The principal (or highest derivative) parts of these evolution equations are given by

$$
\begin{gathered}
\partial_{t} g_{i j} \simeq N^{n} \partial_{n} g_{i j}, \\
\partial_{t} K_{i j} \simeq N^{n} \partial_{n} K_{i j}-N\left[\left(1+2 \gamma_{0}\right) g^{c d} \delta^{n}{ }_{(i} \delta^{b}{ }_{j)}\right. \\
-\left(1+\gamma_{2}\right) g^{n d} \delta^{b}{ }_{i} \delta^{c}{ }_{j)}-\left(1-\gamma_{2}\right) g^{b c} \delta^{n}{ }_{i} \delta^{d}{ }_{j)} \\
\left.+g^{n b} \delta_{i}^{c} \delta^{d}{ }_{j}+2 \gamma_{1} g^{n[b} g^{d] c} g_{i j}\right] \partial_{n} D_{b c d},
\end{gathered}
$$

$$
\begin{aligned}
\partial_{t} D_{k i j} \simeq & N^{n} \partial_{n} D_{k i j}-N\left[\delta^{n}{ }_{k} \delta_{i}^{b} \delta^{c}{ }_{j}-\frac{1}{2} \gamma_{3} g^{n b} g_{k(i} \delta^{c}{ }_{j)}\right. \\
& -\frac{1}{2} \gamma_{4} g^{n b} g_{i j} \delta^{c}{ }_{k}+\frac{1}{2} \gamma_{3} g^{b c} g_{k(i} \delta^{n}{ }_{j)} \\
& \left.+\frac{1}{2} \gamma_{4} g^{b c} g_{i j} \delta^{n}{ }_{k}\right] \partial_{n} K_{b c},
\end{aligned}
$$

where $\simeq$ indicates that terms algebraic in the fields are not shown explicitly. This form of the equations assumes that the lapse density,

$$
Q=\log \left(\mathrm{Ng}^{-\gamma_{0}}\right),
$$

and the shift $N^{i}$ are specified a priori as functions of the coordinates rather than being evolved as independent dynamical fields. The parameter ${ }^{1} \gamma_{0}$ that appears in these equations is part of the definition of the lapse density, Eq. (4), while the parameters $\gamma_{1}, \gamma_{2}, \gamma_{3}$, and $\gamma_{4}$ were introduced by adding multiples of the constraints to the standard Arnowitt-Deser-Misner (ADM) form of the evolution equations (see KST [29]). This form of the Einstein equations is a quasilinear first-order system that can be written more abstractly as

$$
\partial_{t} u^{\alpha}+A_{\beta}^{k \alpha} \partial_{k} u^{\beta}=F^{\alpha},
$$

where $u^{\alpha}=\left\{g_{i j}, K_{i j}, D_{k i j}\right\}$ is the 30 dimensional vector of dynamical fields, and the quantities $A^{k \alpha}{ }_{\beta}$ and $F^{\alpha}$ depend on $u^{\alpha}$ but not its derivatives $\partial_{k} u^{\alpha}$. In this paper the Greek indices $\alpha$ and $\beta$ are used to label elements of this space of dynamical fields.

Boundary conditions for hyperbolic evolution systems like Eq. (5) are imposed on the incoming characteristic fields of the system at each boundary point. These characteristic fields are defined as follows. Given a direction field $n_{k}$ (e.g. the outward directed unit vector normal to the boundary) we define the left eigenvectors $e^{\hat{\alpha}}{ }_{\alpha}$ of the characteristic matrix $n_{k} A_{\beta}^{k \alpha}$ by

$$
e_{\alpha}^{\hat{\alpha}} n_{k} A_{\beta}^{k \alpha}=v_{(\hat{\alpha})} e_{\beta}^{\hat{\alpha}},
$$

where $v_{(\hat{\alpha})}$ is the eigenvalue (also called the characteristic speed). Greek indices with hats, like $\hat{\alpha}$, label the various eigenvectors and corresponding eigenvalues of the system. There is no summation over $\hat{\alpha}$ in Eq. (6). These eigenvectors form a complete set in any strongly hyperbolic system of equations, so the matrix $e^{\hat{\alpha}}{ }_{\alpha}$ is invertible in this case. The projections of the dynamical fields $u^{\alpha}$ onto these characteristic eigenvectors are called the characteristic fields $u^{\hat{\alpha}}$ of the system:

$$
u^{\hat{\alpha}}=e_{\beta}^{\hat{\alpha}} u^{\beta} .
$$

Boundary conditions must be imposed on any character-

\footnotetext{
${ }^{1}$ The parameters $\gamma_{0}$ to $\gamma_{4}$ used here are related to the parameters of Ref. [29] by $\gamma_{0}=\sigma, \gamma_{1}=\gamma, \gamma_{2}=\zeta, \gamma_{3}=\eta$, and $\gamma_{4}=$ $\chi$.
} 
istic field having a negative characteristic speed $v_{(\hat{\alpha})}<0$ (i.e., an incoming field) at a particular boundary point.

The characteristic fields of the KST system are the collection of fields $u^{\hat{\alpha}}=\left\{g_{i j}, Z^{1}, Z_{i}^{2}, Z_{i}^{3}, Z_{i}^{4}, Z_{i j}^{5}, Z_{k i j}^{6}\right.$, $\left.U^{1 \pm}, U_{i}^{2 \pm}, U^{3 \pm}, U_{i j}^{4 \pm}\right\}$. The definitions and explicit expressions for these fields are given in Appendix A. The characteristic fields $U^{1 \pm}, U_{i}^{2 \pm}$, and $U^{3 \pm}$ have characteristic speeds $\pm v_{1}, \pm v_{2}$, and $\pm v_{3}$ respectively relative to the hypersurface orthogonal observers, where

$$
\begin{gathered}
v_{1}^{2}=2 \gamma_{0}, \\
v_{2}^{2}=\frac{1}{8} \gamma_{3}\left(1-3 \gamma_{2}-4 \gamma_{0}\right)-\frac{1}{4} \gamma_{4}\left(1+6 \gamma_{0}\right), \\
v_{3}^{2}=\frac{1}{2}\left(1+2 \gamma_{1}\right)\left(2-\gamma_{3}+2 \gamma_{4}\right)-\frac{1}{2} \gamma_{2} \gamma_{3} .
\end{gathered}
$$

The characteristic fields $U_{i j}^{4 \pm}$ have speeds \pm 1 , and $\left\{g_{i j}, Z^{1}, Z_{i}^{2}, Z_{i}^{3}, Z_{i}^{4}, Z_{i j}^{5}, Z_{k i j}^{6}\right\}$ have characteristic speed zero relative to the hypersurface orthogonal observers.

The KST evolution system is strongly hyperbolic if and only if the characteristic fields are linearly independent. This is the case if $v_{1} \neq 0, v_{2} \neq 0, v_{3} \neq 0$, and $v_{1} \neq v_{3}$. The system is also strongly hyperbolic for $v_{1}=v_{3} \neq 0$ and $1+3 v_{1}^{2}=4 v_{2}^{2}$. In the strongly hyperbolic case the fundamental characteristic fields have 30 independent components: the spatial metric $g_{i j}$ (with six independent components), five scalars $\left\{Z^{1}, U^{1 \pm}, U^{3 \pm}\right\}$, five transverse (to $n^{k}$ ) vectors $\left\{Z_{i}^{2}, Z_{i}^{3}, Z_{i}^{4}, U_{i}^{2 \pm}\right\}$ (which have a total of ten independent components), three transverse-traceless second-rank tensors $\left\{Z_{i j}^{5}, U_{i j}^{4 \pm}\right\}$ (which have a total of seven independent components, since $U_{i j}^{4 \pm}$ is symmetric while $Z_{i j}^{5}$ is not), and one transverse-traceless third-rank tensor $Z_{k i j}^{6}$ (which has a total of two independent components). The KST system also admits a positive-definite symmetrizer matrix $S_{\alpha \beta}$ (and so is symmetric hyperbolic) under fairly weak conditions on the KST parameters, which are discussed in detail in Appendix B of Ref. [32].

The characteristic speeds relative to the coordinate frame, i.e., the eigenvalues $v_{(\hat{\alpha})}$ of Eq. (6), are $\pm N v_{1}-$ $n_{k} N^{k}, \pm N v_{2}-n_{k} N^{k}, \pm N v_{3}-n_{k} N^{k}$, and $\pm N-n_{k} N^{k}$ for the fields $U^{1 \pm}, U_{i}^{2 \pm}, U^{3 \pm}$, and $U_{i j}^{4 \pm}$ respectively. And the speeds are $-n_{k} N^{k}$ for the "zero-speed" fields $\left\{g_{i j}, Z^{1}, Z_{i}^{2}, Z_{i}^{3}, Z_{i}^{4}, Z_{i j}^{5}, Z_{k i j}^{6}\right\}$. Boundary conditions must be supplied for each incoming characteristic field, i.e., for each field whose coordinate characteristic speed is negative at a particular boundary point. The fields $U^{1-}$, $U_{i}^{2-}, U^{3-}$, and $U_{i j}^{4-}$ will be ingoing at most timelike boundaries; the zero-speed fields $\left\{g_{i j}, Z^{1}, Z_{i}^{2}, Z_{i}^{3}\right.$, $\left.Z_{i}^{4}, Z_{i j}^{5}, Z_{k i j}^{6}\right\}$ will be ingoing at any boundary where the shift $N^{i}$ is directed out of the computational domain, i.e., whenever $n_{k} N^{k}>0$. Boundary conditions must be formulated therefore for each of the fields $\left\{g_{i j}, Z^{1}, Z_{i}^{2}\right.$, $\left.Z_{i}^{3}, Z_{i}^{4}, Z_{i j}^{5}, Z_{k i j}^{6}, U^{1-}, U_{i}^{2-}, U^{3-}, U_{i j}^{4-}\right\}$.

\section{CONSTRAINT EVOLUTION SYSTEM}

Boundary conditions capable of preventing the influx of constraint violations cannot be formulated without a complete understanding of how the constraints propagate. The constraints associated with the (vacuum) Einstein evolution system are

$$
\begin{gathered}
\left.C=\frac{1}{2}{ }^{[(3)} R-K_{i j} K^{i j}+K^{2}\right], \\
C_{i}=\nabla_{j} K_{i}^{j}-\nabla_{i} K, \\
C_{k i j}=\partial_{k} g_{i j}-2 D_{k i j}, \\
C_{k l i j}=2 \partial_{[k} D_{l] i j} .
\end{gathered}
$$

Here $C$ is the Hamiltonian constraint, $C_{i}$ is the momentum constraint, while $C_{k i j}$ and $C_{k l i j}$ are auxiliary constraints associated with the introduction of the dynamical field $D_{k i j}$ needed to make the KST system first order. All of these constraint fields are zero for the physical solutions of the (vacuum) Einstein evolution system. Note that $C_{k i j}$ and $C_{k l i j}$ are not completely independent: there is a secondclass constraint $C_{k l i j}=\partial_{[l} C_{k] i j}$. However, $C_{k i j}$ and $C_{k l i j}$ must both be retained and treated as independent in order to write the evolution of the constraints as a closed firstorder hyperbolic system, which is the goal of this section.

The evolution of the constraints is completely determined by the evolution of the dynamical fields of the principal evolution system. Using the KST evolution equations, Eqs. (1)-(3), and the definitions of the constraints, Eqs. (11)-(14), it is straightforward to show that the principal parts of the constraint evolution equations are

$$
\begin{gathered}
\partial_{t} C \simeq N^{k} \partial_{k} C-\frac{1}{2}\left(2-\gamma_{3}+2 \gamma_{4}\right) N g^{i j} \partial_{i} C_{j} \\
\partial_{t} C_{i} \simeq N^{k} \partial_{k} C_{i}-\left(1+2 \gamma_{1}\right) N \partial_{i} C \\
+\frac{1}{2} N g^{k l} g^{a b}\left[\left(1-\gamma_{2}\right) \partial_{k} C_{l a b i}+\left(1+\gamma_{2}\right) \partial_{k} C_{a i l b}\right. \\
\left.-\left(1+2 \gamma_{0}\right) \partial_{k} C_{l i a b}\right] \\
\partial_{t} C_{k i j} \simeq N^{k} \partial_{k} C_{k i j} \\
\partial_{t} C_{k l i j} \simeq N^{m} \partial_{m} C_{k l i j}+\frac{1}{2} \gamma_{3} N\left(g_{j[l} \partial_{k]} C_{i}+g_{i[l} \partial_{k]} C_{j}\right) \\
+\gamma_{4} N g_{i j} \partial_{[k} C_{l]} .
\end{gathered}
$$

More abstractly, the constraints satisfy a quasilinear evolution system of the form

$$
\partial_{t} c^{A}+A_{B}^{k A} \partial_{k} c^{B}=F_{B}^{A} c^{B},
$$

where $c^{A}=\left\{C, C_{i}, C_{k i j}, C_{k l i j}\right\}$ are the constraints defined in Eqs. (11)-(14), $A^{k A}{ }_{B}$ depends on the dynamical fields 
$u^{\alpha}$, and $F_{B}^{A}$ depends on $u^{\alpha}$ and $\partial_{k} u^{\alpha}$. We use upper case Latin indices such as $A$ and $B$ to label the constraint fields. If the constraint evolution system is hyperbolic, then Eq. (19) guarantees that the constraints will vanish everywhere if they are zero at some initial time, and if boundary conditions are chosen to prevent the influx of constraints through timelike boundaries.

In order to determine whether this constraint evolution system is hyperbolic, we evaluate the characteristic fields associated with Eq. (19). It is straightforward to show that the fields $c^{\hat{A}}=\left\{C_{k i j}, Z^{7}, Z_{i}^{8}, Z_{i}^{9}, Z_{i j}^{10}, Z_{i j k l}^{12}, U_{i}^{5 \pm}, U^{6 \pm}\right\}$ are characteristic constraint fields, where the individual components of $c^{\hat{A}}$ are defined by

$$
\begin{gathered}
Z^{7}=\gamma_{3} C-\left(2-\gamma_{3}+2 \gamma_{4}\right) n^{k} n^{l} C_{k l}^{2}, \\
Z_{i}^{8}=n^{k} P_{i}^{l}\left[\gamma_{4} C_{k l}^{1}-\left(\gamma_{3}+3 \gamma_{4}\right) n^{a} n^{b} C_{k l a b}\right], \\
Z_{i}^{9}=n^{k} P_{i}^{l}\left(3 C_{k l}^{1}+2 C_{k l}^{2}-7 n^{a} n^{b} C_{k l a b}\right), \\
Z_{i j}^{10}=P^{k}{ }_{i} P_{j}^{l} C_{k l}^{1}, \\
Z_{i j k l}^{11}=\left(P_{i j}^{k} P^{l}{ }_{j}-\frac{1}{2} P_{i j} P^{k l}\right) C_{k l}^{2}, \\
-\frac{4}{5} C_{[i j]}^{2} g_{k l}-\frac{3}{15} C_{i j}^{1} g_{k l}-\frac{2}{5}\left(C_{k[i}^{2} C_{k[i} g_{j] l}+C_{l[i}^{1} g_{j] k}\right) \\
-\frac{14}{15}\left(g_{k[i} C_{j] l}^{2}+g_{l[i}^{2} C_{j] k}^{2}\right), \\
\left.U_{i}^{5 \pm} g_{j] k}\right) \\
+n^{k} P_{i}^{l}{ }_{i}\left[\left(1+2 \gamma_{0}\right) C_{k l}^{1}+2 C_{[k l]}^{2}-2 \gamma_{2} C_{(k l)}^{2}\right] \\
+2 v_{2} P_{i}^{l} C_{l}, \\
U^{6 \pm}=\left(1+2 \gamma_{1}\right) C \pm v_{3} n^{k} C_{k}-\gamma_{2} n^{k} n^{l} C_{k l}^{2} .
\end{gathered}
$$

The tensor $P^{l}{ }_{i}$ that appears in Eqs. (20)-(27) is the projection tensor $P_{i}^{l}=\delta^{l}{ }_{i}-n^{l} n_{i}, v_{2}$ and $v_{3}$ are given by Eqs. (9) and (10), and $C_{i j}^{1}$ and $C_{i j}^{2}$ are defined by

$$
\begin{aligned}
& C_{i j}^{1}=g^{k l} C_{i j k l}, \\
& C_{i j}^{2}=g^{k l} C_{k i j l} .
\end{aligned}
$$

The characteristic constraint fields $U_{i}^{5 \pm}$ and $U^{6 \pm}$ have characteristic speeds $\pm v_{2}$ and $\pm v_{3}$ respectively, while the characteristic constraint fields $\left\{C_{k i j}, Z^{7}, Z_{i}^{8}, Z_{i}^{9}, Z_{i j}^{10}\right.$, $\left.Z_{i j}^{11}, Z_{i j k l}^{12}\right\}$ have characteristic speed zero relative to the hypersurface orthogonal observers. The characteristic constraint fields have 40 independent components: $C_{k i j}$ (which has 18 independent components), three scalars $\left\{Z^{7}, U^{6 \pm}\right\}$, four transverse (to $n^{k}$ ) vectors $\left\{Z_{i}^{8}, Z_{i}^{9}, U_{i}^{5 \pm}\right\}$ (which have a total of eight independent components), one antisymmetric transverse second-rank tensor $Z_{i j}^{10}$ (which has one independent component), one transverse-traceless second-rank tensor $Z_{i j}^{11}$ (which has three independent components), and one totally trace-free fourth-rank tensor $Z_{i j k l}^{12}$ (which is antisymmetric in its first two indices, symmetric in its last two indices, and so has seven independent components). These characteristic constraint fields are linearly independent so long as $v_{2} \neq 0$ and $v_{3} \neq 0$. Thus the characteristic constraint evolution equations are strongly hyperbolic whenever the principal evolution system is strongly hyperbolic. In Appendix B we summarize the conditions under which the constraint evolution system is also symmetric hyperbolic.

\section{CONSTRAINT-PRESERVING BOUNDARY CONDITIONS}

The constraint characteristic fields $U_{i}^{5-}$ and $U^{6-}$ are incoming fields at most timelike boundaries, and the fields $\left\{C_{k i j}, Z^{7}, Z_{i}^{8}, Z_{i}^{9}, Z_{i j}^{10}, Z_{i j}^{11}, Z_{i j k l}^{12}\right\}$ are incoming at any boundary where $n_{k} N^{k}>0$. Therefore we must choose boundary conditions on the incoming characteristic fields of the principal evolution system, $u^{\hat{\alpha}}$, in a way that controls these incoming constraint characteristic fields, $c^{\hat{A}}$. We use two different approaches to accomplish this for the KST system. The first approach (introduced by Stewart [17] and developed by Calabrese et al. [22]) reexpresses the incoming characteristic constraint fields, $c^{\hat{A}}$, in terms of the principal characteristic fields, $u^{\hat{\alpha}}$. This results in a set of Neumann-like boundary conditions on certain incoming characteristic fields $u^{\hat{\alpha}}$. These type I boundary conditions are discussed in more detail in Sec. IVA. The second (less general) approach (introduced by Holst et al. [3]) uses a more direct Dirichlet-like boundary condition for certain incoming characteristic fields $u^{\hat{\alpha}}$. These type II boundary conditions are discussed in more detail in Sec. IV B.

We note that it is not possible to use these methods to derive a boundary condition for all of the characteristic fields (of the principal system) that need them. In particular it is not possible to obtain boundary conditions for the fields $Z_{i}^{4}, U^{1-}$, and $U_{i j}^{4-}$ in this way. The boundary conditions for these fields are determined by physical and gauge considerations, which are discussed in detail in Secs. V and VI.

\section{A. Type I boundary conditions}

The incoming constraint characteristic fields $C_{k i j}, Z^{7}$, $Z_{i}^{8}, Z_{i}^{9}, Z_{i j}^{11}, Z_{i j k l}^{12}, U_{i}^{5-}$, and $U^{6-}$ all depend on normal (i.e., perpendicular to the boundary) derivatives of the principal characteristic fields $u^{\hat{\alpha}}$. A straightforward but lengthy calculation shows that these characteristic constraint fields can be expressed as follows:

$$
n^{k} C_{k i j}=d_{\perp} g_{i j}-2 n^{k} D_{k i j},
$$




$$
\begin{aligned}
Z^{7}= & -d_{\perp} Z^{1}-\left(2-\gamma_{3}+2 \gamma_{4}\right) P^{a b} n^{c} n^{d} \partial_{a} D_{c d b} \\
& +\frac{1}{2} \gamma_{3}\left(8 D_{[a b]}{ }^{b} D_{c}{ }^{a c}-D_{a b}{ }^{b} D^{a c}{ }_{c}-K_{a b} K^{a b}+K^{2}\right) \\
& -2 \gamma_{3} P^{a b} g^{c d} \partial_{a} D_{[b c] d}+\frac{1}{2} \gamma_{3} D_{a b c}\left(3 D^{a b c}-2 D^{c a b}\right),
\end{aligned}
$$

$$
\begin{aligned}
Z_{i}^{8}= & d_{\perp} Z_{i}^{2}-\left[\gamma_{4} g^{a b}-\left(\gamma_{3}+3 \gamma_{4}\right) n^{a} n^{b}\right] n^{c} P^{d}{ }_{i} \partial_{d} D_{c a b}, \\
Z_{i}^{9}= & d_{\perp} Z_{i}^{3}-\left[\left(3 g^{a b}-7 n^{a} n^{b}\right) P^{d}{ }_{i}-2 P^{a}{ }_{i} P^{b d}\right] n^{c} \partial_{d} D_{c a b}, \\
Z_{i j}^{11}= & d_{\perp} Z_{i j}^{5} \\
& -\left(P^{a}{ }_{i} P^{b}{ }_{j}-\frac{1}{2} P_{i j} P^{a b}\right)\left(g^{c d} \partial_{a} D_{c b d}-P^{c d} \partial_{c} D_{a b d}\right), \\
& n^{d} P_{k i j}^{c a b} Z_{d c a b}^{12}=d_{\perp} Z_{k i j}^{6}-n^{c} P_{k i j}^{d a b} \partial_{d} D_{c a b}, \\
U_{i}^{5-}= & -d_{\perp} U_{i}^{2-}+2 v_{2}\left(P^{j k} P_{i}^{l}-g^{j l} P^{k}{ }_{i}\right) \partial_{k} K_{j l} \\
& +2 v_{2}\left(g^{a j} g^{b k} P^{c}{ }_{i}+g^{c j} g^{a b} P^{k}{ }_{i}\right. \\
& \left.-2 g^{c a} g^{b j} P^{k}{ }_{i}\right) D_{c a b} K_{j k}-\left[\left(1+\gamma_{2}\right) n^{m} g^{l n}\right. \\
& \left.-\left(1+2 \gamma_{0}\right) n^{l} g^{m n}\right] P_{i}^{k} \partial_{k} D_{l m n}-\left[\left(1-\gamma_{2}\right) n^{l} P^{m}{ }_{i}\right. \\
& \left.-\left(1+\gamma_{2}\right) n^{m} P^{l}{ }_{i}\right] P^{k n} \partial_{k} D_{l m n},
\end{aligned}
$$

$$
\begin{aligned}
U^{6-}= & d_{\perp} U^{3-}-v_{3} n^{l} P^{j k} \partial_{k} K_{j l}-\gamma_{2} P^{k n} n^{l} n^{m} \partial_{k} D_{l m n} \\
& +\left(1+2 \gamma_{1}\right)\left(2 g^{m[l} P^{n] k} \partial_{k} D_{l m n}+g^{i[j} g^{a] b} K_{i j} K_{a b}\right) \\
& -v_{3}\left(g^{a j} g^{b k} n^{c}+g^{c j} g^{a b} n^{k}-2 g^{c a} g^{b j} n^{k}\right) D_{c a b} K_{j k} \\
& -\frac{1}{2}\left(1+2 \gamma_{1}\right)\left(g^{k c} g^{i j} g^{a b}+2 g^{k a} g^{i b} g^{j c}\right. \\
& \left.+8 g^{k[i} g^{a] j} g^{c b}-3 g^{k c} g^{i a} g^{j b}\right) D_{k i j} D_{c a b},
\end{aligned}
$$

where $P_{k i j}^{c a b}$ is defined by Eq. (A15), and the quantities $d_{\perp} g_{i j}, d_{\perp} Z^{1}, d_{\perp} Z_{i}^{2}, d_{\perp} Z_{i}^{3}, d_{\perp} Z_{i j}^{5}, d_{\perp} Z_{k i j}^{6}, d_{\perp} U_{i}^{2-}$, and $d_{\perp} U^{3-}$ are components of $d_{\perp} u^{\hat{\alpha}}$, the characteristic projections of the normal derivatives of the dynamical fields.
These projected normal derivatives are defined by

$$
d_{\perp} u^{\hat{\alpha}} \equiv e^{\hat{\alpha}} n^{k} \partial_{k} u^{\beta}
$$

We note that Eqs. (30)-(37) express these incoming characteristic constraint fields, $c^{\hat{A}}$, in terms of the normal derivative of one of the principal characteristic fields, $u^{\hat{\alpha}}$, plus terms that depend on derivatives tangent to the boundary and terms that are algebraic in the fields.

The simplest type I constraint-preserving boundary conditions are obtained by setting the incoming components of the characteristic constraint fields to zero. Using Eqs. (30)(37), these expressions become boundary conditions for the normal derivatives of the incoming dynamical fields $d_{\perp} g_{i j}, d_{\perp} Z^{1}, d_{\perp} Z_{i}^{2}, d_{\perp} Z_{i}^{3}, d_{\perp} Z_{i j}^{5}, d_{\perp} Z_{k i j}^{6}, d_{\perp} U_{i}^{2-}$, and $d_{\perp} U^{3-}$ :

$$
d_{\perp} g_{i j}=2 n^{k} D_{k i j}
$$

$$
\begin{aligned}
& d_{\perp} Z^{1}=-\left(2-\gamma_{3}+2 \gamma_{4}\right) P^{a b} n^{c} n^{d} \partial_{a} D_{c d b} \\
&+\frac{1}{2} \gamma_{3}\left(8 D_{[a b]}{ }^{b} D_{c}{ }^{a c}-D_{a b}{ }^{b} D^{a c}{ }_{c}-K_{a b} K^{a b}+K^{2}\right) \\
&-2 \gamma_{3} P^{a b} g^{c d} \partial_{a} D_{[b c] d} \\
&+\frac{1}{2} \gamma_{3} D_{a b c}\left(3 D^{a b c}-2 D^{c a b}\right), \\
& d_{\perp} Z_{i}^{2}= {\left[\gamma_{4} g^{a b}-\left(\gamma_{3}+3 \gamma_{4}\right) n^{a} n^{b}\right] n^{c} P^{d}{ }_{i} \partial_{d} D_{c a b}, } \\
& d_{\perp} Z_{i}^{3}= {\left[\left(3 g^{a b}-7 n^{a} n^{b}\right) P^{d}{ }_{i}-2 P^{a}{ }_{i} P^{b d}\right] n^{c} \partial_{d} D_{c a b}, } \\
& d_{\perp} Z_{i j}^{5}=\left(P^{a}{ }_{i} P^{b}{ }_{j}-\frac{1}{2} P_{i j} P^{a b}\right)\left(g^{c d} \partial_{a} D_{c b d}-P^{c d} \partial_{c} D_{a b d}\right) \\
& d_{\perp} Z_{k i j}^{6}=n^{c} P_{k i j}^{d a b} \partial_{d} D_{c a b},
\end{aligned}
$$

$$
\begin{aligned}
d_{\perp} U_{i}^{2-}= & 2 v_{2}\left(P^{j k} P_{i}^{l}-g^{j l} P^{k}{ }_{i}\right) \partial_{k} K_{j l}+2 v_{2}\left(g^{a j} g^{b k} P_{i}^{c}\right. \\
& \left.+g^{c j} g^{a b} P^{k}{ }_{i}-2 g^{c a} g^{b j} P^{k}{ }_{i}\right) D_{c a b} K_{j k} \\
& -\left[\left(1+\gamma_{2}\right) n^{m} g^{l n}-\left(1+2 \gamma_{0}\right) n^{l} g^{m n}\right] P^{k}{ }_{i} \partial_{k} D_{l m n} \\
& -\left[\left(1-\gamma_{2}\right) n^{l} P^{m}{ }_{i}-\left(1+\gamma_{2}\right) n^{m} P_{i}^{l}{ }_{i} P^{k n} \partial_{k} D_{l m n},\right.
\end{aligned}
$$

More general type I boundary conditions of this type can also be obtained by setting the incoming constraint characteristic fields to multiples of the corresponding outgoing fields: i.e., by setting $U_{i}^{5-}=\mu_{5} U_{i}^{5+}$ and $U^{6-}=\mu_{6} U^{6+}$. These boundary conditions can therefore be generalized by adding the terms $\mu_{5} U_{i}^{5+}$ and $-\mu_{6} U^{6+}$ to the right sides of Eqs. (45) and (46) respectively.

$$
\begin{aligned}
& +v_{3}\left(g^{a j} g^{b k} n^{c}+g^{c j} g^{a b} n^{k}-2 g^{c a} g^{b j} n^{k}\right) D_{c a b} K_{j k} \\
& +\frac{1}{2}\left(1+2 \gamma_{1}\right)\left(g^{k c} g^{i j} g^{a b}+2 g^{k a} g^{i b} g^{j c}+8 g^{k[i} g^{a] j} g^{c b}-3 g^{k c} g^{i a} g^{j b}\right) D_{k i j} D_{c a b} .
\end{aligned}
$$


We point out that the combination of constraints,

$$
Z_{i}^{9}-15 P^{a}{ }_{i} N^{b} n^{c} n^{d} Z_{a b c d}^{12}=16 P^{a[c} P^{d]}{ }_{i} n^{b} \partial_{d} D_{c a b},
$$

involves no normal derivatives of $D_{c a b}$. Thus, arbitrary multiples of this combination of constraints could be added to the type I boundary conditions for $Z_{i}^{2}, Z_{i}^{3}$ and $U_{i}^{2-}$ in Eqs. (41), (42), and (45) without changing the basic structure of these conditions at all. We find that the addition of these terms does change the stability of numerical evolutions. But at present we have not found a systematic way to optimize the addition of these extra constraint fields (short of brute force numerical testing), so the numerical evolutions presented here use the simplest analytical forms given in Eqs. (41), (42), and (45).

We find it convenient to impose these type I constraintpreserving boundary conditions via the Bjørhus method [33] (which we commonly use in our numerical code) in the following way. The characteristic projection of the principal evolution system can be written as

$$
d_{t} u^{\hat{\alpha}}+v_{(\hat{\alpha})} d_{\perp} u^{\hat{\alpha}}=e_{\beta}^{\hat{\alpha}}\left(-A_{\alpha}^{k \beta} P_{k}^{i} \partial_{i} u^{\alpha}+F^{\beta}\right),
$$

where $d_{t} u^{\hat{\alpha}}$ represents the characteristic projection of the time derivative of the dynamical field:

$$
d_{t} u^{\hat{\alpha}} \equiv e^{\hat{\alpha}}{ }_{\beta} \partial_{t} u^{\beta} .
$$

The terms on the right side of Eq. (48) depend on the boundary values of the dynamical fields and the derivatives of these fields tangent to the boundary surface. We leave these terms unchanged. However, we replace the term $d_{\perp} u^{\hat{\alpha}}$ on the left side of Eq. (48) by its desired value $d_{\perp} u_{\mathrm{bc}}^{\hat{\alpha}}$. For the fields $g_{i j}, Z^{1}, Z_{i}^{2}, Z_{i}^{3}, Z_{i j}^{5}, U_{i}^{2-}$ and $U^{3-}$, $d_{\perp} u_{\mathrm{bc}}^{\hat{\alpha}}$ is given by Eqs. (39)-(46).

This method of imposing these boundary conditions can be implemented numerically in a simple and elegant way. Let us define the quantity

$$
D_{t} u^{\hat{\alpha}} \equiv e_{\beta}^{\hat{\alpha}}\left(-A_{\alpha}^{k \beta} \partial_{k} u^{\alpha}+F^{\beta}\right),
$$

which is just the characteristic projection of the right side of the full evolution equation, without any modifications for boundary conditions. At the boundary, Eq. (48) then takes the form

$$
d_{t} u^{\hat{\alpha}}=D_{t} u^{\hat{\alpha}}+v_{(\hat{\alpha})}\left(d_{\perp} u^{\hat{\alpha}}-d_{\perp} u_{\mathrm{bc}}^{\hat{\alpha}}\right) .
$$

In this expression, $d_{\perp} u_{\mathrm{bc}}^{\hat{\alpha}}$ represents the desired values of the tangential derivatives at the boundary, and $d_{\perp} u^{\hat{\alpha}}$ denotes the actual values of the tangential derivatives as defined in Eq. (38). When $d_{\perp} u^{\hat{\alpha}}$ is expressed in terms of the constraints on the boundary using Eqs. (30)-(37), and when $d_{\perp} u_{\mathrm{bc}}^{\hat{\alpha}}$ is determined from Eqs. (39)-(46), then the term $d_{\perp} u^{\hat{\alpha}}-d_{\perp} u_{\mathrm{bc}}^{\hat{\alpha}}$ simplifies considerably. Thus, in particular, the boundary conditions for $g_{i j}, Z^{1}, Z_{i}^{2}, Z_{i}^{3}, Z_{i j}^{5}$, $Z_{k i j}^{6}, U_{i}^{2-}$ and $U^{3-}$ can be written as

$$
d_{t} g_{i j}=D_{t} g_{i j}-n_{k} N^{k} n^{l} C_{l i j}
$$

$$
\begin{gathered}
d_{t} Z^{1}=D_{t} Z^{1}+n_{k} N^{k} Z^{7}, \\
d_{t} Z_{i}^{2}=D_{t} Z_{i}^{2}-n_{k} N^{k} Z_{i}^{8}, \\
d_{t} Z_{i}^{3}=D_{t} Z_{i}^{3}-n_{k} N^{k} Z_{i}^{9}, \\
d_{t} Z_{i j}^{5}=D_{t} Z_{i j}^{5}-n_{k} N^{k} Z_{i j}^{11}, \\
d_{t} Z_{k i j}^{6}=D_{t} Z_{k i j}^{6}-n_{m} N^{m} n^{d} P_{k i j}^{c a b} Z_{d c a b}^{12}, \\
d_{t} U_{i}^{2-}=D_{t} U_{i}^{2-}+\left(N v_{2}+n_{k} N^{k}\right)\left(U_{i}^{5-}-\mu_{5} U_{i}^{5+}\right), \\
d_{t} U^{3-}=D_{t} U^{3-}-\left(N v_{3}+n_{k} N^{k}\right)\left(U^{6-}-\mu_{6} U^{6+}\right) .
\end{gathered}
$$

The $D_{t} u^{\hat{\alpha}}$ that appear in these expressions are to be evaluated using Eq. (50), while the constraint fields $C_{k i j}, Z^{7}$, $Z_{i}^{8}, Z_{i}^{9}, Z_{i j}^{11}, Z_{k l i j}^{12}, U_{i}^{5-}$ and $U^{6-}$ are to be evaluated numerically using their definitions in Eqs. (13), (20)-(22), and (24)-(27) respectively.

\section{B. Type II boundary conditions}

A second type of constraint-preserving boundary condition can be imposed on those characteristic fields that represent various projections of the field $P^{k}{ }_{l} D_{k i j}$. The influx of constraint violations that could cause $P^{k}{ }_{l} D_{k i j}$ to differ from $P^{k}{ }_{l} \partial_{k} g_{i j} / 2$ can be prevented by enforcing the equality of these fields on the relevant boundaries. This is a Dirichlet-like boundary condition that sets $P^{k}{ }_{l} D_{k i j}=$ $P^{k}{ }_{l} \partial_{k} g_{i j} / 2$. This type of constraint-preserving boundary condition has been used successfully in the scalar-wave system by Holst et al. [3]. The characteristic fields $Z_{i}^{2}, Z_{i}^{3}$, $Z_{i j}^{5}$, and $Z_{k i j}^{6}$ are composed entirely of various components of $P_{l}^{k} D_{k i j}$, which represent the derivatives of $g_{i j}$ that are tangent to the boundary. These type II boundary conditions can be imposed using the Bjørhus method by setting

$$
\begin{gathered}
d_{t} Z_{i}^{2}=\frac{1}{2}\left[\gamma_{4} P^{a b}-\left(\gamma_{3}+2 \gamma_{4}\right) n^{a} n^{b}\right] P_{i}^{c} \partial_{c} \partial_{t} g_{a b}, \\
d_{t} Z_{i}^{3}=\frac{1}{2}\left[\left(3 P^{a b}-4 n^{a} n^{b}\right) P_{i}^{c}-2 P_{i}^{a} P^{b c}\right] \partial_{c} \partial_{t} g_{a b}, \\
d_{t} Z_{i j}^{5}=\frac{1}{4}\left(2 P^{c}{ }_{i} P^{a}{ }_{j}-P_{i j} P^{c a}\right) n^{b} \partial_{c} \partial_{t} g_{a b}, \\
d_{t} Z_{k i j}^{6}=\frac{1}{2} P_{k i j}^{c a b} \partial_{c} \partial_{t} g_{a b},
\end{gathered}
$$

at any boundary where these fields are incoming. The quantity $\partial_{c} \partial_{t} g_{a b}$ that appears on the right sides of Eqs. (60)-(63) is to be evaluated by taking the indicated tangential spatial derivatives of the right side of Eq. (52). These expressions are obtained using the expressions for $Z_{i}^{2}, Z_{i}^{3}, Z_{i j}^{5}$, and $Z_{k i j}^{6}$ from Eqs. (A2), (A3), (A5), and (A6), 
and replacing the terms $\partial_{t} D_{c a b}$ by $\partial_{c} \partial_{t} g_{a b} / 2$. We note that these fields $Z_{i}^{2}, Z_{i}^{3}, Z_{i j}^{5}$ and $Z_{k i j}^{6}$ can also be controlled using the type I boundary conditions described in Sec. IVA. We have tested these type II boundary conditions numerically, and find the results to be essentially identical to the results described in Sec. VIII for tests with type I boundary conditions.

\section{PHYSICAL BOUNDARY CONDITIONS}

In this section we derive boundary conditions for the physical components of the dynamical fields, $U_{i j}^{4-}$, which represent the real gravitational-wave degrees of freedom of the system, at least asymptotically. Our strategy is based on the proposal of Bardeen and Buchman [18], and similar ideas used by Reula and Sarbach [34] in the context of the Maxwell system. These ideas are adapted to the general 3D Einstein system by analyzing the dynamics of the Weyl tensor as determined by the Bianchi identities. We then infer boundary conditions on the dynamical fields of the KST system that control the incoming radiative parts of the Weyl tensor. We begin by following the usual practice of decomposing the Weyl tensor into electric and magnetic parts,

$$
\begin{gathered}
E_{\mu \nu}=C_{\mu \sigma \nu \tau} T^{\sigma} T^{\tau}, \\
B_{\mu \nu}=\frac{1}{2} C_{\mu \omega \sigma \tau} \epsilon_{\nu \rho}^{\sigma \tau} T^{\omega} T^{\rho},
\end{gathered}
$$

where $T^{\mu}$ represents a timelike unit vector. [In this paper letters from the latter part of the Greek alphabet $(\mu, \nu$, etc.) represent four-dimensional spacetime coordinate indices.] In our analysis here we assume that $T^{\mu}$ is orthogonal to the $t=$ constant spacelike hypersurfaces of the standard $3+1$ decomposition of the geometry. In this case we can express the electric and magnetic parts of the Weyl tensor in terms of standard $3+1$ quantities:

$$
\begin{gathered}
E_{i j}=R_{i j}+K K_{i j}-K_{i}{ }^{k} K_{k j}, \\
B_{i j}=\left(\nabla_{k} K_{l i}\right) \epsilon_{j}^{k l},
\end{gathered}
$$

where $R_{i j}, K_{i j}, \nabla_{i}$ and $\epsilon_{i j k}$ represent the three-dimensional Ricci tensor, the extrinsic curvature, the $3 \mathrm{D}$ spatial covariant derivative, and the $3 \mathrm{D}$ totally antisymmetric tensor, respectively. These electric and magnetic parts of the Weyl tensor are symmetric and traceless in any vacuum spacetime.

The Weyl tensor satisfies the Bianchi identities,

$$
0=\nabla_{[\sigma} C_{\tau \omega] \mu \nu},
$$

in any vacuum spacetime, where here $\nabla_{\mu}$ represents the $4 \mathrm{D}$ covariant derivative, and these equations imply a system of evolution equations for the Weyl tensor. The $3+1$ representation of the principal parts of these evolution equations can be written [35] in the form

$$
\begin{gathered}
\partial_{t} E_{i j}-N^{k} \partial_{k} E_{i j} \simeq-N\left[\partial_{k} B_{l(i}\right] \epsilon_{j)}{ }^{k l}, \\
\partial_{t} B_{i j}-N^{k} \partial_{k} B_{i j} \simeq N\left[\partial_{k} E_{l(i]}\right] \epsilon_{j)}{ }^{k l},
\end{gathered}
$$

which is reminiscent of the Maxwell system. The characteristic fields of this vacuum Weyl tensor evolution system are

$$
\begin{gathered}
Z^{13}=n^{a} n^{b} E_{a b}, \\
Z^{14}=n^{a} n^{b} B_{a b}, \\
U_{i}^{7 \pm}=P_{i}^{a} n^{b} E_{a b} \mp \epsilon_{i}^{a b} n_{b} n^{c} B_{a c}, \\
U_{i j}^{8 \pm}=\left(P^{(a}{ }_{i} P_{j}^{b)}-\frac{1}{2} P_{i j} P^{a b}\right)\left(E_{a b} \mp \epsilon_{a}{ }^{c d} n_{d} B_{c b}\right),
\end{gathered}
$$

where $n^{k}$ is a spatial unit vector. The characteristic fields $Z^{13}$ and $Z^{14}$ have characteristic speed zero, $U_{i}^{7 \pm}$ have speeds $\pm 1 / 2$, and $U_{i j}^{8 \pm}$ have speeds \pm 1 relative to the hypersurface orthogonal observers. These fields propagate with coordinate speeds $-n_{k} N^{k}, \pm N / 2-n_{k} N^{k}$, and $\pm N-$ $n_{k} N^{k}$ respectively. An equivalent expression for $U_{i j}^{8 \pm}$ in terms of the standard $3+1$ variables is

$$
\begin{aligned}
U_{i j}^{8 \pm}= & \left(P^{a}{ }_{i} P^{b}{ }_{j}-\frac{1}{2} P_{i j} P^{a b}\right)\left(R_{a b}+K K_{a b}-K_{a}{ }^{c} K_{c b}\right. \\
& \left.\mp n^{c} \nabla_{c} K_{a b} \pm n^{c} \nabla_{(a} K_{b) c}\right) .
\end{aligned}
$$

The vacuum characteristic fields $U_{i j}^{8+}$ and $U_{i j}^{8-}$ are proportional to the Newman-Penrose [36] components of the Weyl tensor $\Psi_{4}$ and $\Psi_{0}$, respectively.

The true gravitational-wave degrees of freedom are represented by the fields $U_{i j}^{8 \pm}$. The best (gauge invariant) way to impose boundary conditions on the physical degrees of freedom of the Einstein system is therefore to require that the incoming part of this field has prescribed values: $U_{i j}^{8-}=\left.U_{i j}^{8-}\right|_{\mathrm{bc}}$ at the boundaries. This condition is equivalent to fixing the Newman-Penrose component $\Psi_{0}=\left.\Psi_{0}\right|_{\text {bc }}$ on the boundary. Since $U_{i j}^{8-}$ falls to zero as $r^{-5}$ in an asymptotically flat spacetime [36], it is reasonable to set $U_{i j}^{8-}=0$ as an approximate physical no-incoming-wave boundary condition, even for a boundary located at a finite radius. This boundary condition, $U_{i j}^{8-}=0$, would produce discontinuities in the incoming waves unless $U_{i j}^{8-}$ were exactly zero in the initial data. Therefore instead we freeze $U_{i j}^{8-}$ to its (small) initial value by setting $\left.U_{i j}^{8-}\right|_{\mathrm{bc}}=U_{i j}^{8-}(t=0)$. This condition guarantees continuity and therefore produces smoother numerical evolutions. In the limit of boundaries with larger and larger radius, the $U_{i j}^{8-}=0$ and the $\left.U_{i j}^{8-}\right|_{\mathrm{bc}}=U_{i j}^{8-}(t=0)$ boundary conditions will be equivalent in an asymptotically flat spacetime.

In order to write the boundary condition on $U_{i j}^{8-}$ as a condition on the principal dynamical fields, we first express $U_{i j}^{8-}$ in terms of the principal characteristic fields using Eq. (75): 


$$
\begin{aligned}
U_{i j}^{8-}= & d_{\perp} U_{i j}^{4-}-\gamma_{2} Z_{(i j)}^{11}-\left[P^{(a}{ }_{i} P_{j}^{b)}-\frac{1}{2} P_{i j} P^{a b}\right]\left\{n^{c} \partial_{a} K_{b c}+2 \gamma_{2} P^{c d} \partial_{[a} D_{c] b d}+\gamma_{2} n^{c} n^{d} \partial_{a} D_{c b d}+2 P^{c d} \partial_{c} D_{[d a] b}\right. \\
& +2 g^{c d} \partial_{a} D_{[b c] d}-K K_{a b}+K_{a c} K_{b}^{c}+\left(2 D^{c d}{ }_{c}-D^{d c}{ }_{c}\right)\left(2 D_{a b d}-D_{d a b}\right)+4 D^{c d}{ }_{a} D_{[d c] b}-D_{a}{ }^{c d} D_{b c d} \\
& \left.+2 n^{c} K_{[a}^{d} D_{c] b d}+4 n^{c} D_{[b d][c} K_{a]}{ }^{d}\right\} .
\end{aligned}
$$

In deriving this expression, we have used the fact that the constraint characteristic field $Z_{i j}^{11}$ can be expressed in terms of $d_{\perp} Z_{i j}^{5}$ using Eq. (34). Setting $U_{i j}^{8-}=\left.U_{i j}^{8-}\right|_{\mathrm{bc}}\left(\right.$ and $\left.Z_{(i j)}^{11}=0\right)$ gives us the desired Neumann-like boundary condition for the principal characteristic field $U_{i j}^{4-}$ :

$$
\begin{aligned}
d_{\perp} U_{i j}^{4-}= & \left.U_{i j}^{8-}\right|_{b c}+\left[P_{i}^{(a} P_{j}^{b)}-\frac{1}{2} P_{i j} P^{a b}\right]\left\{n^{c} \partial_{a} K_{b c}+2 \gamma_{2} P^{c d} \partial_{[a} D_{c] b d}+\gamma_{2} n^{c} n^{d} \partial_{a} D_{c d b}+2 P^{c d} \partial_{c} D_{[d a] b}\right. \\
& +2 g^{c d} \partial_{a} D_{[b c] d}-K K_{a b}+K_{a c} K_{b}^{c}+4 D^{c d}{ }_{a} D_{[d c] b}+\left(2 D^{c d}{ }_{c}-D^{d c}{ }_{c}\right)\left(2 D_{a b d}-D_{d a b}\right)-D_{a}{ }_{a} d D_{b c d} \\
& \left.+2 n^{c} K^{d}{ }_{[a} D_{c] b d}+4 n^{c} D_{[b d][c} K_{a]}{ }_{a}\right\} .
\end{aligned}
$$

This physical boundary condition can be imposed numerically with the same technique used for the type I constraint-preserving boundary conditions discussed in Sec. IVA. In particular we set

$$
\begin{aligned}
d_{t} U_{i j}^{4-}= & D_{t} U_{i j}^{4-} \\
& -\left(N+n_{k} N^{k}\right)\left[U_{i j}^{8-}-\left.U_{i j}^{8-}\right|_{\mathrm{bc}}+\gamma_{2} Z_{(i j)}^{11}\right] .
\end{aligned}
$$

The term $D_{t} U_{i j}^{4-}$ is to be evaluated using Eq. (50), while $Z_{i j}^{11}$ and $U_{i j}^{8-}$ are to be evaluated numerically using Eqs. (24) and (75) respectively.

\section{GAUGE-FIXING BOUNDARY CONDITIONS}

Next we turn our attention to finding boundary conditions for the two fields $U^{1-}$ and $Z_{i}^{4}$ that were not fixed by either the constraint-preserving boundary conditions in Sec. IV or the physical boundary conditions in Sec. V. Since these fields are not fixed by the constraints or by physical considerations, they must be gauge fields in effect. Boundary conditions on $U^{1-}$ and $Z_{i}^{4}$ should be chosen in a way that is consistent with the gauge conditions. In the evolution system used here, we assume that the lapse density and the shift are frozen to their initial values. One reasonable boundary condition for these gauge fields then is simply to freeze them at their initial values on the boundaries:

$$
\begin{gathered}
d_{t} U^{1-}=0, \\
d_{t} Z_{i}^{4}=0 .
\end{gathered}
$$

We have also found that a useful boundary condition for $U^{1-}$ can be obtained from one of the standard equations used to fix the lapse: $\partial_{t} K=0$. Expressing $\partial_{t} K$ in terms of the time derivatives of the principal characteristic fields, we find

$$
\begin{aligned}
\partial_{t} K= & g^{i j} \partial_{t} K_{i j}-K^{i j} \partial_{t} g_{i j}, \\
= & \frac{3-q}{4 v_{3}}\left(d_{t} U^{3+}+d_{t} U^{3-}\right) \\
& +\frac{1}{4 v_{1}}\left(d_{t} U^{1+}+d_{t} U^{1-}\right)-K^{i j} \partial_{t} g_{i j} .
\end{aligned}
$$

[The parameter $q$ that appears in this expression is the ratio of characteristic speeds defined in Eq. (A14).] Setting $\partial_{t} K=0$ therefore provides a boundary condition for the incoming characteristic field $U^{1-}$ :

$$
\begin{aligned}
d_{t} U^{1-}= & -d_{t} U^{1+}-\frac{v_{1}(3-q)}{v_{3}}\left(d_{t} U^{3+}+d_{t} U^{3-}\right) \\
& +4 v_{1} K^{i j} \partial_{t} g_{i j} .
\end{aligned}
$$

The quantities $d_{t} U^{3-}$ and $\partial_{t} g_{i j}$ on the right side of Eq. (82) are evaluated with the appropriate boundary expressions for these quantities. We find that using Eq. (82) in evolutions of black-hole spacetimes is fairly effective in controlling the growth of perturbations in $U^{1-}$ with low spherical-harmonic indices. But at the same time using this boundary condition causes unstable (and nonconvergent) growth of perturbations with large sphericalharmonic indices. We solve this problem numerically by applying this boundary condition only after applying a filter that removes all spherical-harmonic components above $\ell=2$ from Eq. (82) at the boundary. Thus we use Eq. (82) for the $\ell \leq 2$ spherical-harmonic components of this equation, and a regular freezing boundary condition, Eq. (79), for the $\ell>2$ spherical-harmonic components.

Finally, we note that boundary conditions for $U^{1-}$ and $Z_{i}^{4}$ can also be obtained from the " $\Gamma$-freezing" condition that is often used to determine the shift vector [37,38]. We found that these $\Gamma$-freezing boundary conditions on $U^{1-}$ and $Z_{i}^{4}$ are not as effective as Eqs. (80) and (82) in controlling the growth of instabilities in these gauge fields. And since the equations for the $\Gamma$-freezing forms of these boundary conditions are quite lengthy, we do not reproduce them here. 


\section{NUMERICAL METHODS}

In this section we describe briefly the numerical methods used to compute the simulations presented below. All our numerical computations are performed using a multidomain pseudospectral collocation method. Our numerical methods are essentially the same as those we applied previously to evolution problems with scalar fields [3,39], with the Maxwell system [14], and the Einstein system $[29,40-42]$.

\section{A. Spectral collocation method}

The computational domain for the single black-hole evolutions described in Sec. VIII is a spherical shell extending from some $r_{\min }$ (just inside the black-hole event horizon) to some maximum value $r_{\max }$. This domain may also be subdivided into one or more spherical-shell subdomains. Consider a single one of these subdomains that extends from radius $r_{\min }^{\prime}$ to $r_{\max }^{\prime}$. Given a system of partial differential equations

$$
\partial_{t} u^{\alpha}(\mathbf{x}, t)=\mathcal{F}^{\alpha}\left[u(\mathbf{x}, t), \partial_{i} u(\mathbf{x}, t)\right],
$$

where $u^{\alpha}$ is a collection of dynamical fields, the solution $u^{\alpha}(\mathbf{x}, t)$ on this subdomain is expressed as a timedependent linear combination of $N$ spatial basis functions $\phi_{k}(\mathbf{x})$ :

$$
u_{N}^{\alpha}(\mathbf{x}, t)=\sum_{k=0}^{N-1} \tilde{u}_{k}^{\alpha}(t) \phi_{k}(\mathbf{x}) .
$$

We expand each Cartesian component of each tensor in terms of the basis functions $T_{n}(\rho) Y_{\ell m}(\theta, \varphi)$, where $Y_{\ell m}(\theta, \varphi)$ are spherical harmonics and $T_{n}(\rho)$ are Chebyshev polynomials with

$$
\rho=\frac{2 r-r_{\max }^{\prime}-r_{\min }^{\prime}}{r_{\max }^{\prime}-r_{\min }^{\prime}} .
$$

The spherical coordinates $(r, \theta$, and $\varphi)$ used in these spectral expansions are related to the pseudo-Cartesian coordinates used to evaluate the components of tensor fields (e.g. $g_{x x}, K_{y z}, D_{x y z}, \ldots$ ) by the usual transformation $x=r \sin \theta \sin \varphi, y=r \sin \theta \cos \varphi$, and $z=r \cos \theta$. In these spectral expansions we keep Chebyshev polynomials $T_{n}(\rho)$ with $n$ up to some finite $N_{r}$, and spherical harmonics $Y_{\ell m}(\theta, \varphi)$ with $\ell$ up to some finite $\ell_{\max }$. For each $\ell$, we keep all $|m| \leq \ell$. The values of $N_{r}$ and $\ell_{\max }$ determine our radial and angular resolution, and we vary these in order to perform convergence tests. Spatial derivatives are evaluated analytically using the known derivatives of the basis functions:

$$
\partial_{i} u_{N}^{\alpha}(\mathbf{x}, t)=\sum_{k=0}^{N-1} \tilde{u}_{k}^{\alpha}(t) \partial_{i} \phi_{k}(\mathbf{x}) .
$$

Associated with the basis functions is a set of $N_{c}$ collocation points $\mathbf{x}_{i}$. Given spectral coefficients $\tilde{u}_{k}^{\alpha}(t)$, the function values at the collocation points $u^{\alpha}\left(\mathbf{x}_{i}, t\right)$ are computed using Eq. (84). Conversely, the spectral coefficients are obtained by the inverse transform

$$
\tilde{u}_{k}^{\alpha}(t)=\sum_{i=0}^{N_{c}-1} w_{i} u_{N}^{\alpha}\left(\mathbf{x}_{i}, t\right) \phi_{k}\left(\mathbf{x}_{i}\right),
$$

where $w_{i}$ are weights specific to the choice of basis functions and collocation points. Thus it is straightforward to transform between the spectral coefficients $\tilde{u}_{k}^{\alpha}(t)$ and the function values at the collocation points $u_{N}^{\alpha}\left(\mathbf{x}_{i}, t\right)$. The partial differential equation, Eq. (83), is now rewritten using Eqs. (84)-(87) as a set of ordinary differential equations for the function values at the collocation points,

$$
\partial_{t} u_{N}^{\alpha}\left(\mathbf{x}_{i}, t\right)=G_{i}^{\alpha}\left[u_{N}\left(\mathbf{x}_{j}, t\right)\right],
$$

where $G_{i}^{\alpha}$ depends on $u_{N}^{\alpha}\left(\mathbf{x}_{j}, t\right)$ for all $j$. We integrate this system of ordinary differential equations, Eq. (88), in time using a fourth-order Runge-Kutta algorithm. Boundary conditions are incorporated into the right side of Eq. (88) using the technique of Bjørhus [33]. For the evolutions reported here the time step is typically chosen to be about 1.5 times the distance between the closest collocation points in order that the Courant-Friedrichs-Lewy stability limit remains satisfied.

We use no filtering on the radial basis functions, but apply a rather complicated filtering rule for the angular functions. When evaluating the right side of Eq. (88), we set to zero the coefficients of the terms with $\ell \geq \ell_{\max }-3$ in the tensor spherical-harmonic expansions of the dynamical-field components (i.e. the $g_{i j}, K_{i j}$, and $D_{k i j}$ components) of this equation. This filtering method eliminates a certain type of angular instability that arises because differentiation mixes the various spherical-harmonic indices in the spectral expansions of the Cartesian components of tensors.

\section{B. Multiple subdomains}

Under many circumstances it is advantageous to divide the computational domain into multiple subdomains. This subdivision allows faster calculations by spreading the computational load over multiple processors. It also allows the use of different spectral resolutions in different parts of the computational domain, thus making it possible to concentrate computational resources in areas where they are most needed. We use the spectral collocation method described in Sec. VII A for each computational subdomain. An additional complication with multiple subdomains is that only some of the subdomain boundaries are external, while some are just internal boundaries that separate subdomains. Appropriate boundary conditions must nevertheless be specified on each boundary of each subdomain. For the computations described here we impose conditions on each subdomain boundary using the Bjørhus [33] method, in which $d_{t} u^{\hat{\alpha}}$ [see Eq. (49)] is specified for each ingoing 
characteristic field (i.e., for each $\hat{\alpha}$ with $v_{(\hat{\alpha})}<0$ ). Noningoing fields do not need and are not given boundary conditions. For external boundaries (those without neighboring subdomains) the incoming $d_{t} u^{\hat{\alpha}}$ are computed according to some externally imposed boundary condition, for example, our new constraint-preserving or physical boundary conditions. For internal boundaries, the values of all incoming $d_{t} u^{\hat{\alpha}}$ are simply copied from the corresponding outgoing $d_{t} u^{\hat{\alpha}}$ in the neighboring subdomain. At a black-hole excision boundary $\boldsymbol{v}_{(\hat{\alpha})}>0$ for all $\hat{\alpha}$, so no boundary condition is required there on any of the dynamical fields.

For the simulations described here, we subdivide our computational domain into a set of concentric sphericalshell subdomains. We choose the constants $N_{r}$ and $\ell_{\max }$ (that specify the spectral resolution of each subdomain) to have the same values in all the subdomains; this makes it easier to achieve load balancing when multiple processors are used. We concentrate the numerical resolution where it is needed in our solutions by allowing spherical shells with varying thickness: for fixed radial resolution $N_{r}$, thin shells achieve higher resolution than thick shells.

\section{Residual evaluators}

We use three different computational diagnostics to help us evaluate the accuracy and stability of the numerical simulations described in Sec. VIII. The first and simplest diagnostic just measures the difference between a numerical solution and the exact solution of the equations. This is possible whenever the exact solution to the problem is known, as in our evolutions of unperturbed black-hole spacetimes. We measure the deviation of the numerical solution from the exact solution quantitatively by evaluating the energy norm of the difference between the two solutions:

$$
(\delta E)^{2}=\int S_{\alpha \beta}\left(u^{\alpha}-u_{0}^{\alpha}\right)\left(u^{\beta}-u_{0}^{\beta}\right) \sqrt{g} d^{3} x .
$$

Here $u^{\alpha}$ denotes the numerical solution, $u_{0}^{\alpha}$ the exact solution, and $S_{\alpha \beta}$ the symmetrizer matrix of the hyperbolic evolution system. The symmetrizer $S_{\alpha \beta}$ is a positivedefinite matrix on the space of dynamical fields, which is derived for the KST evolution system studied here in Refs. [32,41]. (We evaluate $S_{\alpha \beta}$ using the exact solution $u_{0}^{\alpha}$.) The energy $\delta E$ is not dimensionless, and consequently it is not clear how to interpret whether a given value means that the solution is a good approximation of the exact solution or not. Therefore we normalize by dividing by the total energy of the exact solution:

$$
E_{0}^{2}=\int S_{\alpha \beta} u_{0}^{\alpha} u_{0}^{\beta} \sqrt{g} d^{3} x
$$

The ratio $\delta E / E_{0}$ is therefore a good dimensionless measure of the accuracy of our numerical solution. When this ratio becomes of order unity, the numerical solution bears little resemblance to the exact solution.

A second, more general, method of measuring the accuracy and stability of our numerical solutions is to monitor the magnitudes of the constraints, $c^{A}=\left\{C, C_{i}, C_{k i j}, C_{k l i j}\right\}$. When the constraints vanish, the solutions to our first-order evolution system, Eqs. (1)-(3), are guaranteed to be solutions of the original Einstein equations as well. Thus, to measure how well our numerical solutions solve the original Einstein system we construct the following measure of the constraints:

$$
\|C\|^{2}=\int\left(C^{2}+C_{i} C^{i}+C_{k i j} C^{k i j}+C_{k l i j} C^{k l i j}\right) \sqrt{g} d^{3} x .
$$

The constraints consist of various derivatives of the dynamical fields $u^{\alpha}$. Therefore we can construct a meaningful dimensionless measure of the constraints by normalizing $\|C\|$ by dividing by a measure of these derivatives:

$$
\|\partial u\|^{2}=\int \sum_{\alpha} g^{i j} \partial_{i} u^{\alpha} \partial_{j} u^{\alpha} \sqrt{g} d^{3} x .
$$

We evaluate the quantities that appear in Eq. (92) (i.e., the tensor components of $u^{\alpha}$ and the partial derivatives) in the global Cartesian-like coordinate system used in our code. The ratio $\|C\| /\|\partial u\|$ is therefore a dimensionless measure of the degree to which our numerical solutions satisfy the original Einstein system. When this quantity becomes of order unity the constraint violations dominate and our numerical solutions no longer accurately represent solutions to the original Einstein equations.

At the continuum level, the vanishing of $\|C\|$ is enough to ensure that the solutions to our first-order evolution system also represent solutions to the original second-order Einstein equations. It is good numerical practice, however, to construct a redundant independent check of how well the original system of differential equations is satisfied by our numerical solutions. So we have constructed a third independent residual evaluator for the second-order form of the Einstein equations. Following the ideas of Choptuik [43], we use our numerical solution for $u^{\alpha}$ to reconstruct the four-dimensional metric:

$$
\begin{aligned}
d s^{2} & =g_{\mu \nu} d x^{\mu} d x^{\nu} \\
& =-N^{2} d t^{2}+g_{i j}\left(d x^{i}+N^{i} d t\right)\left(d x^{j}+N^{j} d t\right) .
\end{aligned}
$$

[Letters from the latter part of the Greek alphabet $(\mu, \nu$, etc.) denote four-dimensional spacetime indices in our Cartesian-like coordinate system.] We evaluate the components of $g_{\mu \nu}$ on the actual time slices used in our evolution code, and then evaluate the time derivatives $\partial_{t} g_{\mu \nu}$ and $\partial_{t}^{2} g_{\mu \nu}$ using standard centered finite-difference expressions for these quantities with seven-point stencils. Given these time derivatives on a certain time slice, we then evaluate the complete set of first and second deriva- 
tives, $\partial_{\sigma} g_{\mu \nu}$ and $\partial_{\sigma} \partial_{\tau} g_{\mu \nu}$, by computing the spatial derivatives using the spectral methods described in Sec. VII A. Finally, we combine these first and second derivatives to determine the four-dimensional Ricci tensor:

$$
\begin{aligned}
R_{\mu \nu}= & -\frac{1}{2} g^{\sigma \tau}\left(\partial_{\sigma} \partial_{\tau} g_{\mu \nu}+\partial_{\mu} \partial_{\nu} g_{\sigma \tau}-2 \partial_{\sigma} \partial_{(\mu} g_{\nu) \tau}\right) \\
& +\frac{1}{4} g^{\sigma \tau} g^{\omega \rho}\left[\partial_{\mu} g_{\sigma \omega} \partial_{\nu} g_{\tau \rho}+4 \partial_{\sigma} g_{\mu \omega} \partial_{[\tau} g_{\rho] \nu}\right. \\
& \left.-\left(\partial_{\omega} g_{\mu \nu}-2 \partial_{(\mu} g_{\nu) \omega}\right)\left(\partial_{\rho} g_{\sigma \tau}-2 \partial_{\sigma} g_{\tau \rho}\right)\right] .
\end{aligned}
$$

The Ricci tensor should vanish for any solution of the vacuum Einstein equations, so this quantity gives us an independent way to measure how well our numerical solution solves the original second-order Einstein equations.

The expression for the Ricci tensor, Eq. (94), has 13 terms (each of which contains contractions): four terms proportional to $\partial_{\sigma} \partial_{\tau} g_{\mu \nu}$, and nine terms proportional to $\partial_{\sigma} g_{\mu \nu}$ (when symmetrizations and antisymmetrizations are expanded out). We construct a positive-definite quantity, $R_{\mu \nu}^{\mathrm{rms}}$, to which the size of $R_{\mu \nu}$ can be compared, by taking the square root of the sum of the squares of these 13 terms (for each $\mu$ and $\nu$ ). We then construct norms of these quantities by integrating them over our spacelike slices:

$$
\begin{gathered}
\|R\|^{2}=\int \sum_{\mu \leq \nu}\left(R_{\mu \nu}\right)^{2} \sqrt{g} d^{3} x, \\
\left\|R^{\mathrm{rms}}\right\|^{2}=\int \sum_{\mu \leq \nu}\left(R_{\mu \nu}^{\mathrm{rms}}\right)^{2} \sqrt{g} d^{3} x .
\end{gathered}
$$

We note that the $R_{\mu \nu}^{\mathrm{rms}}$ and $R_{\mu \nu}$ used to compute these norms are the quasi-Cartesian components of these quantities used in our code. We use the ratio $\|R\| /\left\|R^{\text {rms }}\right\|$ as our third independent measure of the degree to which our numerical solutions satisfy the original Einstein equations. When both $\|R\| /\left\|R^{\text {rms }}\right\|$ and $\|C\| /\|\partial u\|$ are small, we have considerable confidence in the accuracy of our solutions even in cases where the exact solution is not known.

\section{NUMERICAL EVOLUTIONS}

We have studied the efficacy of the boundary conditions proposed in Secs. IV, V, and VI by performing evolutions of various single black-hole spacetimes. These simulations evolve either unperturbed Schwarzschild black holes in Kerr-Schild coordinates,

$$
d s^{2}=-d t^{2}+\frac{2 M}{r}(d t+d r)^{2}+d r^{2}+r^{2} d \Omega^{2},
$$

or fully dynamical black holes that are small perturbations of the Schwarzschild spacetime (but we solve the full nonlinear equations in our code). We express all lengths and times associated with these simulations in units of the bare black-hole mass $M$, even for the perturbed solutions in which the ADM mass exceeds $M$. The computational domains for these evolutions consist of one or more concentric spherical shells that cover the space from $r_{\text {min }}=$ 1.9M (just inside the black-hole event horizon) to some maximum value $r_{\max }$. We have run evolutions using several different $r_{\max }$ in the range $6.9 M \leq r_{\max } \leq 41.9 M$.

The initial data for these evolutions are prepared by applying an odd-parity outgoing quadrupole-wave perturbation with amplitude $4 \times 10^{-3}$ to the Kerr-Schild spatial metric and its time derivative. This outgoing wave pulse is constructed by Teukolsky's method [44] with the generating function $G(r)$ that determines the radial profile of the wave: $G(r)=A e^{-\left(r-r_{0}\right)^{2} / w^{2}}$, where $A=4 \times 10^{-3}, r_{0}=$ $5 M$ and $w=1.5 M$. Then we solve numerically the full nonlinear conformal "thin-sandwich" form of the initial value equations to obtain constraint-satisfying perturbed black-hole initial data (see Pfeiffer et al. [45]). In the resulting initial data set, the ADM energy exceeds the apparent horizon mass by about $10^{-5} \mathrm{M}$. Figure 1 illustrates the initial data for these perturbed black-hole evolutions. The quantity shown in Fig. 1 is a measure of the outgoing gravitational-wave flux, $\sqrt{U_{i j}^{8+} U_{k l}^{8+} g^{i k} g^{j l} / 2}$, defined in Eq. (74). This quantity is equivalent to the Newman-Penrose component of the Weyl tensor $\left|\Psi_{4}\right|$. The outer radius of the computational domain for the initial data shown in Fig. 1 is $r_{\max }=11.9 M$.

For simplicity we use fixed-gauge evolutions: the lapse density $Q$ and the shift $N^{i}$ are set to their initial Kerr-Schild values, Eq. (97), for all times. For the perturbed initial data, we also set $Q$ and $N^{i}$ to the unperturbed analytical KerrSchild values, ignoring the solutions for these fields given by the conformal thin-sandwich initial value equations. Although more sophisticated gauge conditions should result in better long-term evolutions, these simple fixed-

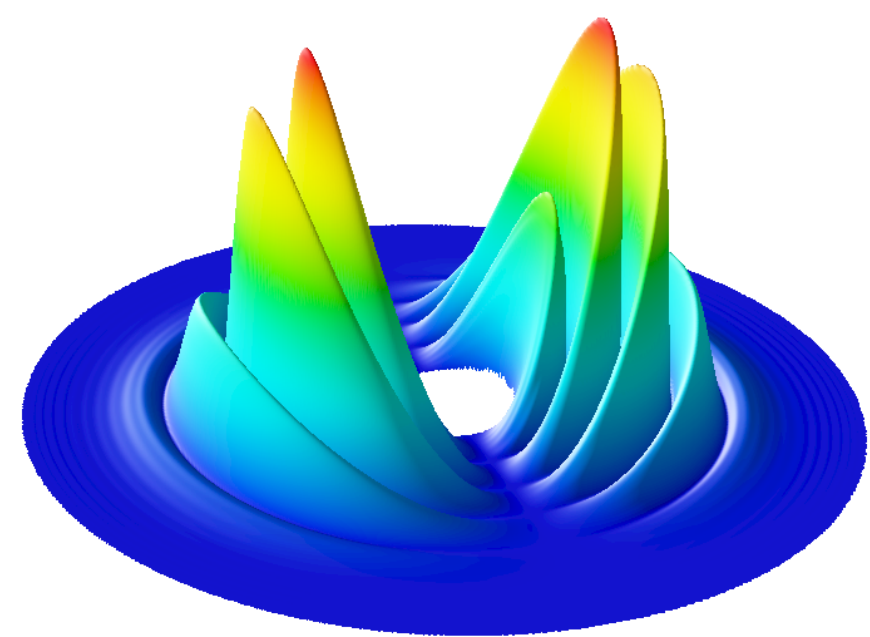

FIG. 1 (color online). Initial data for the perturbed black-hole evolutions. Shown is the Newman-Penrose component of the Weyl tensor $\left|\Psi_{4}\right|$, which measures the outgoing gravitationalwave flux. 
gauge conditions are adequate for the tests of the new constraint-preserving boundary conditions, which are of primary interest here.

The KST evolution system has a number of freely specifiable parameters. We set the KST parameters to the values $\gamma_{0}=0.5, \quad \gamma_{1}=-12, \gamma_{2}=-1, \quad \gamma_{3}=0.16$, and $\gamma_{4}=$ -0.96 for all the evolutions discussed here. This choice of parameters is one of the special cases that leave the parameter $q$ of Eq. (A14) ill defined. In this case the choice of $q$ is arbitrary, and we set $q=1$ in all of the evolutions discussed here (since this yields better performance than $q=0$ ). For these parameter choices, both the fundamental evolution equations and the constraint evolution system are symmetric hyperbolic, and all characteristic speeds (relative to hypersurface orthogonal observers) are either 0 or \pm 1 ; in particular, $v_{1}^{2}=v_{2}^{2}=v_{3}^{2}=1$. The values of the KST parameters used here are the same as those used in previous studies $[41,42]$. However, the current evolution equations are rather different from those used in Refs. [41,42], in which the evolution equations were modified by a kinematical change of variables and by the addition of terms proportional to the constraint $C_{k i j}$. Here we perform no such modification; that is, the kinematical parameters defined in Refs. [29,41,42] are chosen here to have the values $\hat{k}=1, \hat{z}=\hat{a}=\hat{b}=\hat{c}=\hat{d}=\hat{e}=0$. The values of the KST parameters in Refs. [41,42] were chosen to minimize the growth rate of the constraints for evolutions of a single black hole in Painlevé-Gullstrand coordinates. Because we use a rather different set of evolution equations, and because we evolve black holes in KerrSchild coordinates, we do not expect that these parameter values are optimal for our current evolutions.

\section{A. Unperturbed black holes}

In this subsection we describe three numerical tests involving 3D evolutions of unperturbed Schwarzschild black holes. These evolutions use the Schwarzschild spatial metric and extrinsic curvature in Kerr-Schild coordinates, Eq. (97), as initial data. The first and second tests explore the evolutions of these black holes using simple "freezing" boundary conditions. Freezing boundary conditions set the time derivatives of each of the incoming characteristic fields to zero at the boundaries, i.e.,

$$
d_{t} u^{\hat{\alpha}}=0,
$$

for all $\hat{\alpha}$ with $v_{(\hat{\alpha})}<0$ [where $d_{t} u^{\hat{\alpha}}$ is defined in Eq. (49)]. These boundary conditions are known to make the evolution equations mathematically well posed, but they are also known to do a poor job of preventing the influx of constraint violations into the computational domain for fully dynamical time-dependent solutions. Time-dependent evolutions with freezing boundary conditions have been shown to be stable and numerically convergent $[3,14]$ (and so represent real solutions to the continuum evolution equations), but they also violate the constraints and con- sequently do not represent physical solutions to the full constrained evolution system. Despite these limitations, freezing boundary conditions are appropriate and have been used rather successfully to evolve time independent solutions such as the unperturbed black-hole spacetimes considered in this subsection [41,42].

Our first test evolves an unperturbed Schwarzschild black hole on a computational domain extending from $r_{\text {min }}=1.9 M$ to $r_{\max }=41.9 M$. This domain is subdivided into eight subdomains, each of width $5 M$, each using the same angular resolution $\ell_{\max }=21$, and each having the same radial resolution $N_{r}=17,21$ or 26. This angular resolution is much higher than necessary to resolve this spherically symmetric spacetime, but we wanted to verify the stability of our code even for large values of $\ell_{\max }$. Figure 2 shows two measures of the errors in these evolutions: $\|C\| /\|\partial u\|$ and $\delta E / E_{0}$. These results show that our error measures converge toward zero as we increase the radial resolution $N_{r}$. These results also show that our computational methods are capable of evolving unperturbed black-hole solutions for hundreds (if not thousands) of $M$, which is consistent with previous results using a similar evolution system [41,42].

As a second test, we explore the effects of changing the location of the outer boundary of the computational domain, $r_{\max }$, for these unperturbed black-hole evolutions with freezing boundary conditions. Figure 3 shows the constraint violations $\|C\| /\|\partial u\|$ and the energy norms $\delta E / E_{0}$ for evolutions using several different outer radii:

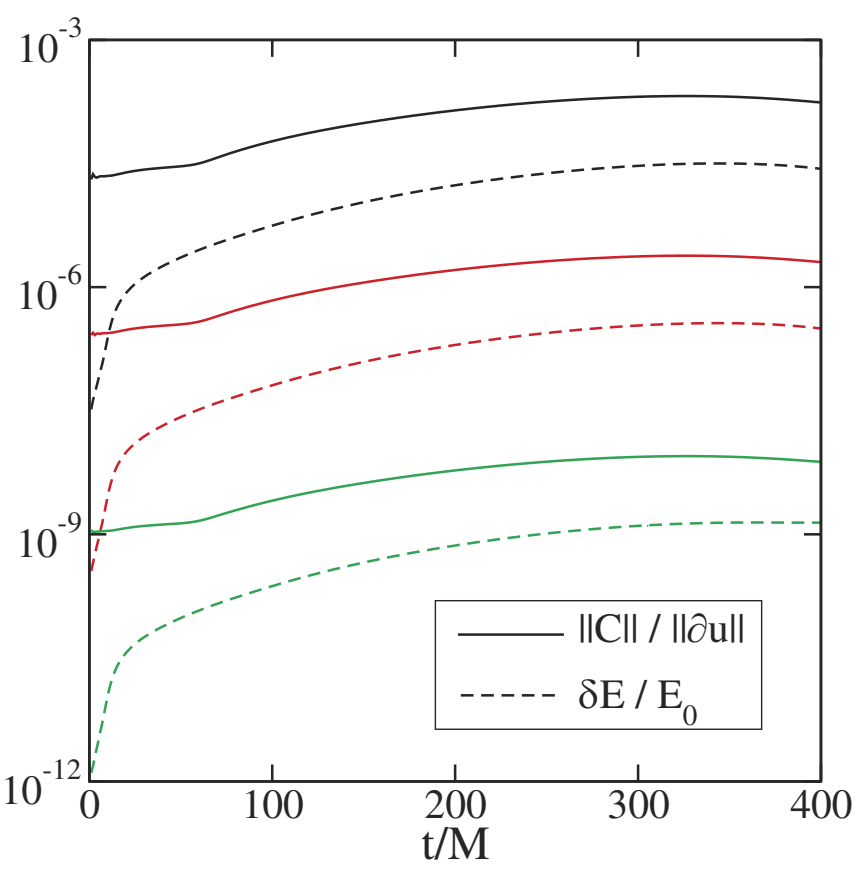

FIG. 2 (color online). Constraint violations and energy norms for unperturbed black-hole evolutions using freezing boundary conditions. The outer boundary is at $r_{\max }=41.9 M$; and three different radial resolutions, $N_{r}=17,21$, and 26, show numerical convergence. 


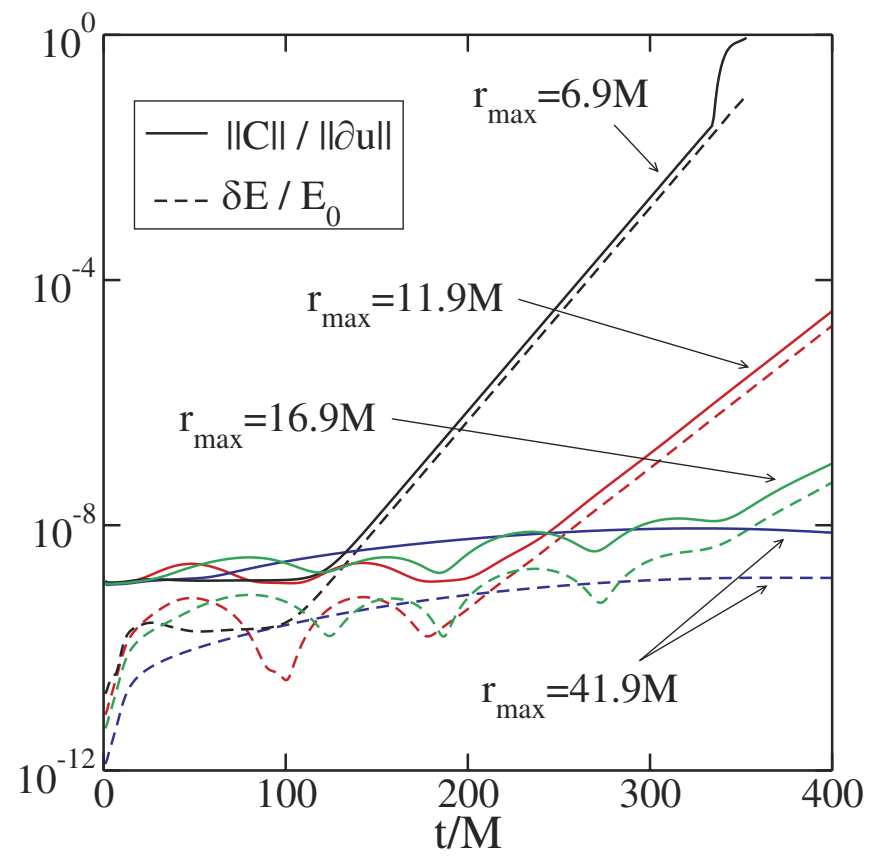

FIG. 3 (color online). Constraint violations and energy norms for unperturbed black-hole evolutions with a range of outer boundary radii, $r_{\max }=6.9 M, 11.9 M, 16.9 M, 41.9 M$. These runs all use $N_{r}=26$ in each subdomain, but use different numbers of subdomains to achieve different $r_{\max }$. They all use freezing boundary conditions, and $\ell_{\max }=21$.

$r_{\max }=6.9 M, 11.9 M, 16.9 M$, and 41.9M. All of these evolutions use the same $\ell_{\max }=21$, and $N_{r}=26$ in each subdomain. Each computational subdomain has width $5 M$, so the number of subdomains is adjusted to achieve the desired $r_{\text {max }}$. Figure 3 shows that these evolutions have an instability that causes $\|C\| /\|\partial u\|$ and $\delta E / E_{0}$ to grow exponentially. This instability becomes weaker as $r_{\max }$ increases; we find (by measuring the growth rate for $r_{\max }=6.9 M, 11.9 M, 16.9 M, 21.9 M$, and $31.9 M$ ) that the growth rate is $M / \tau \approx e^{-r_{\max } / 13 M}$. So this appears to be a constraint-violating instability that is influenced by the location of the outer boundary of the computational domain. Figure 4 demonstrates for the $r_{\max }=11.9 M$ case that these unstable evolutions are numerically convergent, by showing that $\|C\| /\|\partial u\|$ and $\delta E / E_{0}$ approach an exponentially growing solution as $N_{r}$ increases. Figure 4 also shows that the growth rate of the instability is the same for all values of $N_{r}$ in this $r_{\max }=11.9 M$ case. (Numerical convergence and independence of the growth rate with $N_{r}$ is also observed for the other values of $r_{\max }$ that we tested.) We have also verified that the growth rate of this instability does not depend on the angular resolution $\ell_{\max }$ for $7 \leq$ $\ell_{\max } \leq 21$. These checks suggest that this constraintviolating instability is a solution of the continuum differential equations, and is not primarily an artifact of the discrete numerical representation of the equations used here.

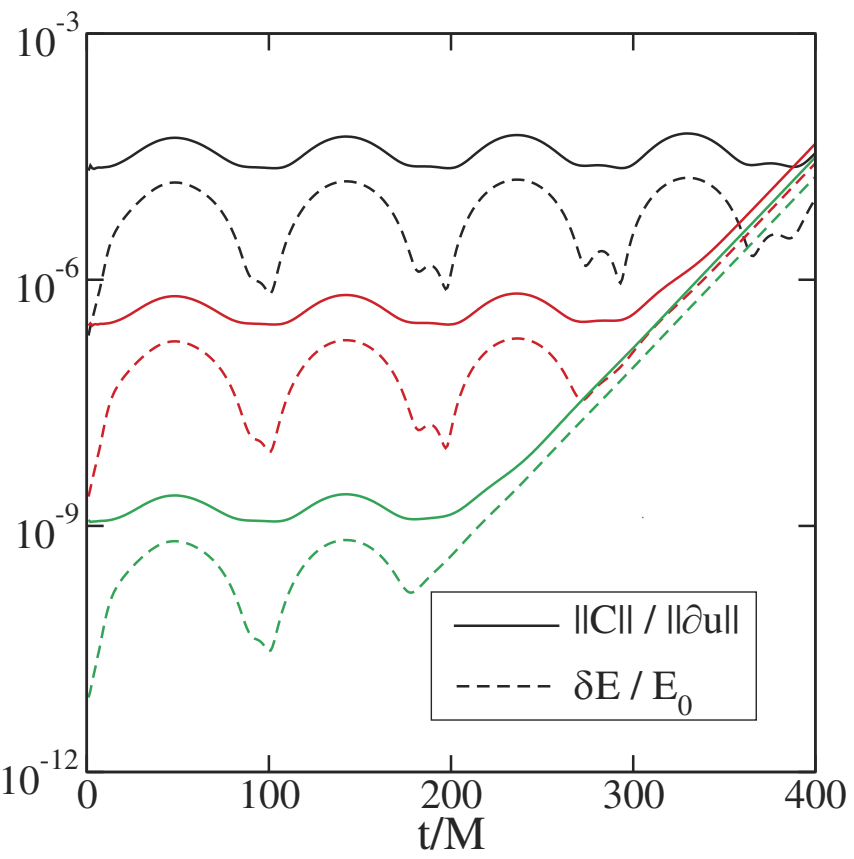

FIG. 4 (color online). Constraint violations and energy norms for an unperturbed black-hole evolution using freezing boundary conditions. The outer boundary is at $r_{\max }=11.9 \mathrm{M}$; and three different radial resolutions, $N_{r}=17,21$, and 26, show numerical convergence to an exponentially growing solution.

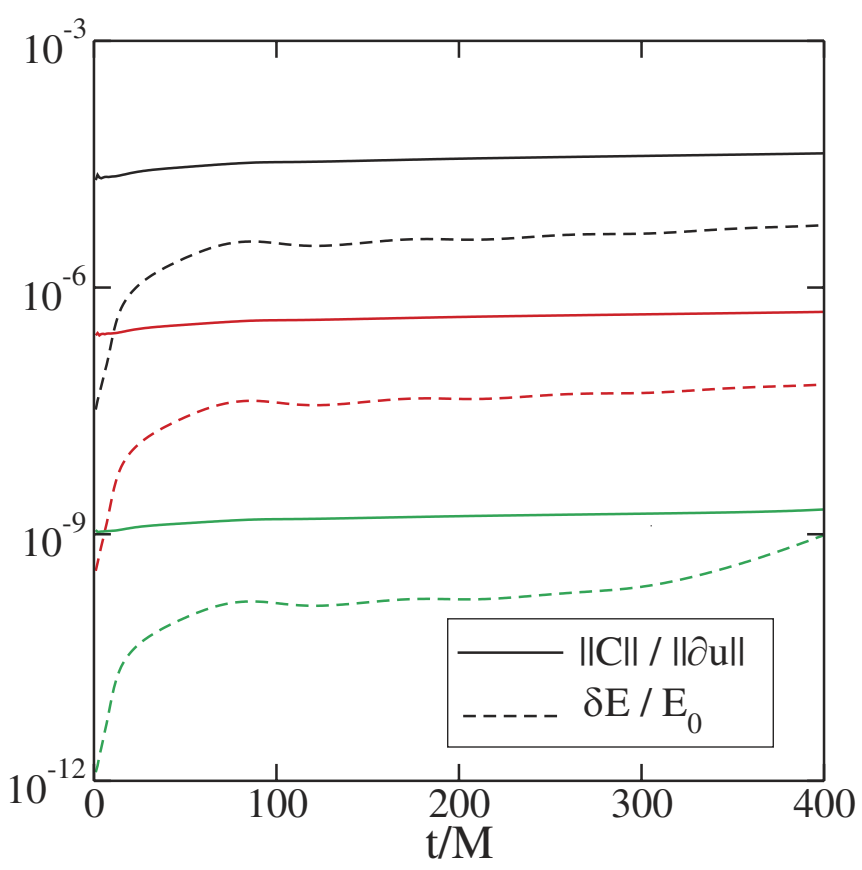

FIG. 5 (color online). Constraint violations and energy norms for unperturbed black-hole evolutions using our new boundary conditions. The outer boundary is at $r_{\max }=41.9 M$; and three different radial resolutions, $N_{r}=17,21$, and 26, show numerical convergence. 
For the third numerical test we use our new boundary conditions to evolve the same unperturbed black-hole initial data on the same computational domains used in the first two tests. These new boundary conditions include the new constraint-preserving boundary conditions, Eqs. (52)(59) with $\mu_{5}=0.75$ and $\mu_{6}=-0.5$, the new physical boundary condition, Eq. (78), and the new gauge boundary conditions, Eqs. (80) and (82). Figure 5 depicts $\|C\| /\|\partial u\|$ and $\delta E / E_{0}$ for evolutions of the unperturbed black-hole initial data on a computational domain with $r_{\max }=41.9 \mathrm{M}$. (Except for boundary conditions, the evolutions in Fig. 5 are identical to those of Fig. 2.) The evolutions of Fig. 5 show that both the constraints and the energy norms of these solutions decrease toward zero as $N_{r}$ increases.

At late times, the highest resolution curve plotted in Fig. 5 shows the beginning of an exponentially growing mode. We call this a gauge mode, because $\delta E / E_{0}$ begins to grow in these solutions without a corresponding growth in the constraints $\|C\| /\|\partial u\|$. This gauge mode grows more rapidly if we move the outer boundary inward, as can be seen in Fig. 6, which shows the analogous evolutions with outer boundary at $r_{\max }=11.9 \mathrm{M}$. The constraints remain roughly constant in the evolutions of Fig. 6 for a considerable amount of time after $\delta E / E_{0}$ begins to grow rapidly. Comparing the results of Fig. 4 with those of Fig. 6, we see that our constraint-preserving boundary conditions do improve the constraint violations in these evolutions, but only slightly. However, this improvement comes at the expense

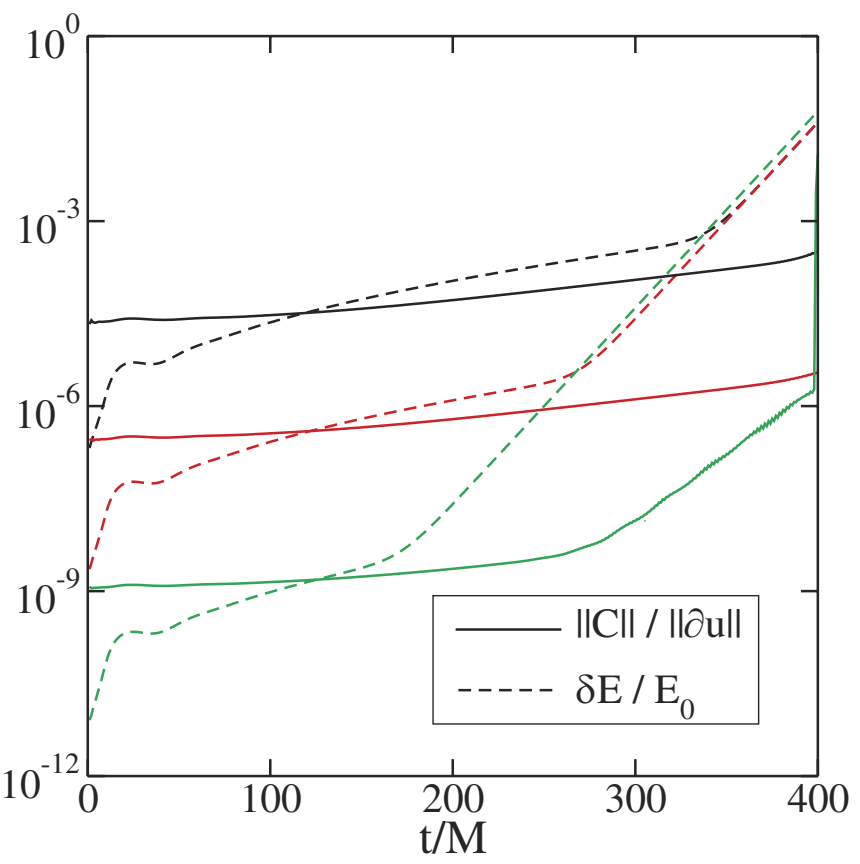

FIG. 6 (color online). Constraint violations and energy norms for an unperturbed black-hole evolution using our new boundary conditions. The outer boundary is at $r_{\max }=11.9 \mathrm{M}$; and three different radial resolutions, $N_{r}=17,21$, and 26, show numerical convergence to an exponentially growing solution. of introducing a new gauge-mode instability. At very late times we see that the highest resolution evolution also shows signs of a constraint-violating instability, although it grows more slowly than the gauge mode. The growth rate of the constraints seen in the highest resolution evolution of Fig. 6 is the same as the growth rate of the instability seen in Fig. 4, to within about 5\%. This suggests that both of these constraint-violating instabilities might be caused primarily by bulk rather than boundary constraint violations.

The gauge-mode instability seen in Figs. 5 and 6 is dominated by its $\ell=3$ spherical-harmonic component. Recall that we impose the gauge-fixing boundary condition, Eq. (82), by filtering out everything but the $\ell \leq 2$ components of $d_{t} U^{1-}$. If we change this filtering to impose Eq. (82) on only the $\ell \leq 1$ components of $d_{t} U^{1-}$, the gauge-mode instability is dominated by its $\ell=2$ spherical-harmonic component, and grows more rapidly than in Figs. 5 and 6. Thus the gauge boundary condition, Eq. (82), does improve the stability of the gauge mode at least to some degree. However, if we impose Eq. (82) without any filtering, the instability grows much faster than the rate seen in Figs. 5 and 6; and this growth rate increases as $\ell_{\max }$ increases, so the evolution becomes nonconvergent. Recall that we use the simplest possible gauge conditions: we set the lapse density $Q$ and the shift $N^{i}$ to their analytic values throughout the evolution. A more

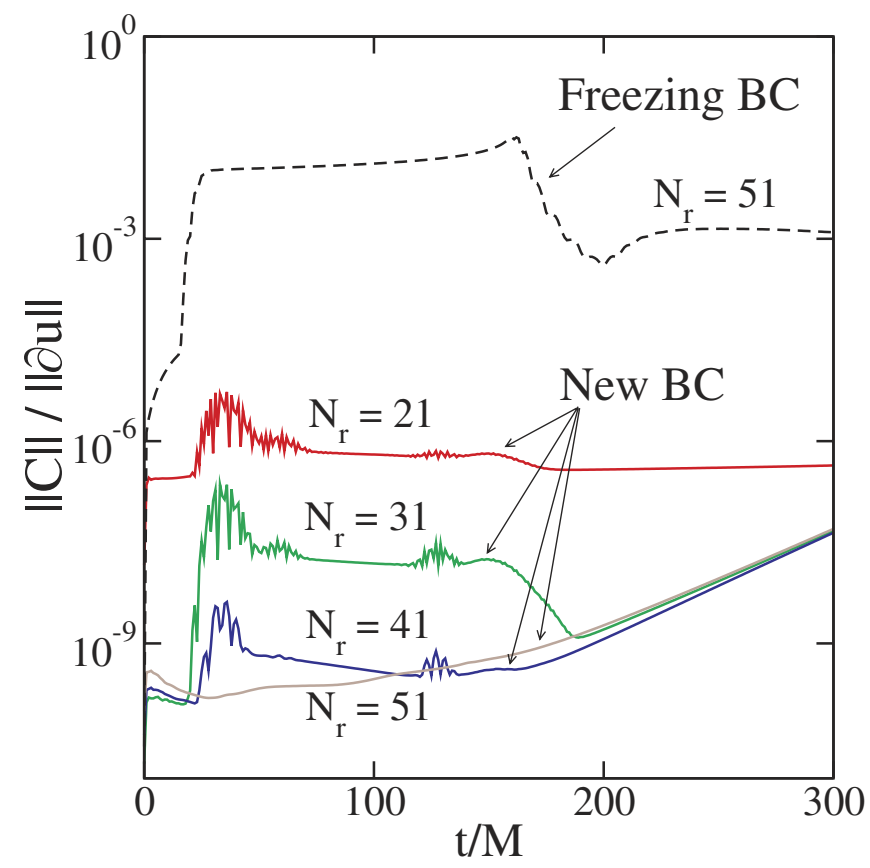

FIG. 7 (color online). Constraint violations for evolutions of perturbed black holes using freezing (dashed curve) and our new boundary conditions (solid curves). Radial resolutions $N_{r}=21$, 31,41 , and 51 are shown for the new boundary conditions, while only $N_{r}=51$ is shown for the freezing case. The outer boundary is at $r_{\max }=21.9$. 
sophisticated treatment of gauge conditions (not just at the boundaries, but throughout the volume) is probably needed to control these unstable gauge modes.

\section{B. Perturbed black holes}

In this subsection we describe 3D numerical evolutions of perturbed black-hole spacetimes. The initial data for these evolutions are discussed at the beginning of Sec. VIII. We evolve these initial data using the same nonlinear equations and evolution methods used in Sec. VIII A for the unperturbed cases. These perturbed black-hole initial data include a short-wavelength gravitational-wave packet, so more radial collocation points are required to achieve an accuracy comparable to that of the unperturbed black-hole cases.

Figure 7 shows the constraint error $\|C\| /\|\partial u\|$ for evolutions of these perturbed black-hole initial data. These simulations are performed on a computational domain with four concentric subdomains, each of width $5 \mathrm{M}$ and $\ell_{\max }=$ 11 , and with outer boundary at $r_{\max }=21.9$. The dashed curve in Fig. 7 shows the results of using the simple freezing boundary conditions, Eq. (98): a large constraint violation is generated at $t \approx 20 M$ when the wave pulse passes through the outer boundary of the computational domain. The size of this constraint violation is comparable to the amplitude of the wave, and does not converge away with increased resolution. Only the highest radial resolution is shown, because the lower resolution curves are almost identical. These simulations with freezing boundary conditions are numerically convergent and do represent solutions to the continuum evolution equations. But they do not satisfy all the equations (i.e., the constraints) of the full constrained evolution system, so they do not represent physical solutions to the Einstein equations. These solutions demonstrate that freezing boundary conditions cannot be used to model the physical gravitational waves in a system after those waves interact with the boundaries.

Also plotted in Fig. 7 are evolutions of the same perturbed black-hole initial data on the same computational domain, but now using our new boundary conditions: the new constraint-preserving boundary conditions, Eqs. (52)(59) with $\mu_{5}=0.75$ and $\mu_{6}=-0.5$, the new physical boundary condition, Eq. (78), and the new gauge boundary conditions, Eqs. (80) and (82). In these cases the constraint violation generated as the wave passes through the outer boundary converges away with increasing radial resolution. The constraint violation is 8 orders of magnitude smaller than with freezing boundary conditions in the highest resolution case. Figure 8 shows an independent measure of how well these numerical evolutions satisfy the original Einstein equations by plotting the average value of the four-dimensional Ricci tensor $\|R\| /\left\|R^{\text {rms }}\right\|$ [see Eq. (95)] for the same simulations shown in Fig. 7. Figure 8 confirms the results seen in Fig. 7: the Einstein equations are violated when the wave hits the boundary with freezing boundary conditions, while the new boundary conditions are quite effective in reducing this violation by many orders of magnitude.

Two interesting features are seen in the evolutions in Figs. 7 and 8 which use the new boundary conditions: first, there is an unstable exponential growth in the highest resolution runs starting at about $t \approx 200 M$, and second, there is some complicated structure starting at about $t \approx$ $20 M$ which is reduced and finally vanishes at the highest radial resolution. Consider first the unstable exponential growth. Figure 9 shows this exponential growth more clearly by displaying the constraint violations for a range $r_{\max }$. The simulations in Fig. 9 have $N_{r}=51$ and $\ell_{\max }=$ 11 in each subdomain, while $r_{\max }$ is varied by adjusting the number of subdomains. However, in order to resolve the small incoming waves reflected off the outer boundary, we need higher resolution near the outer boundary. The subdomains are made narrower therefore for larger $r$, as described below. Figure 9 shows that the exponential growth rate of this constraint-violating instability decreases as $r_{\max }$ increases. The growth rate of this constraint-violating instability is about 1.7 times larger than the growth rate seen in the unperturbed black-hole evolutions of Fig. 3, for each $r_{\max }$. The similarity in size and dependence on the location of the outer boundary suggests that the instabilities in Figs. 3 and 9 may have the same basic cause. If so then this cause is probably bulk generated constraint violations, since the evolutions in Fig. 9 use constraint-preserving boundary conditions.

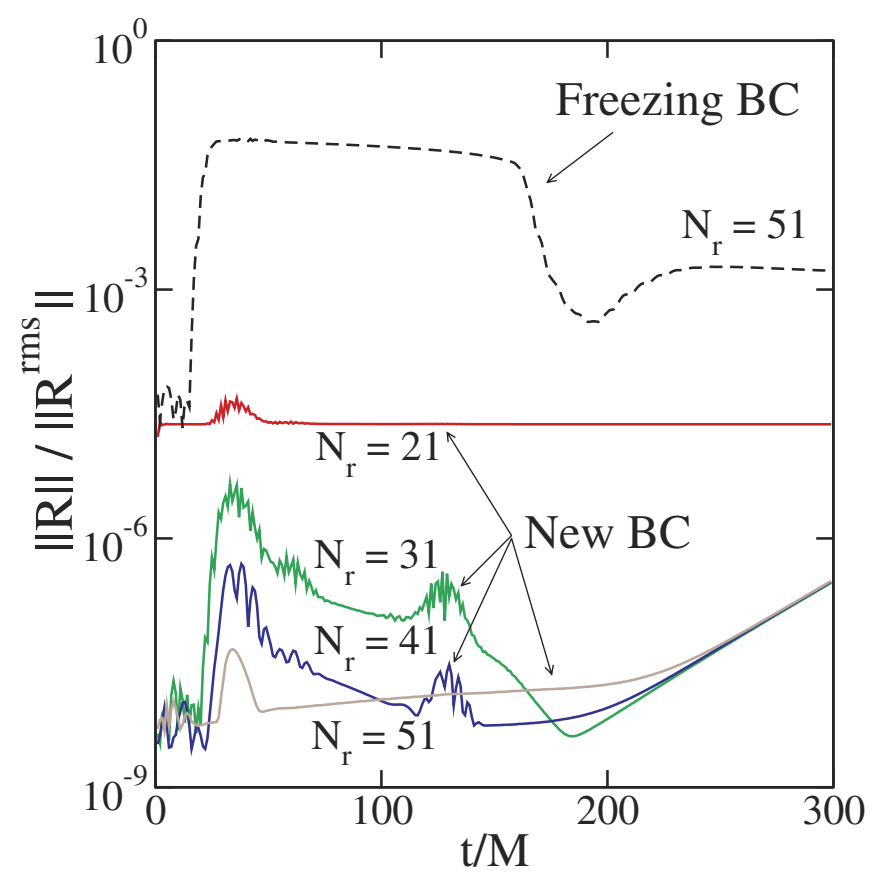

FIG. 8 (color online). Four-dimensional Ricci-tensor residual of Eq. (95) for the perturbed black-hole evolutions shown in Fig. 7. 


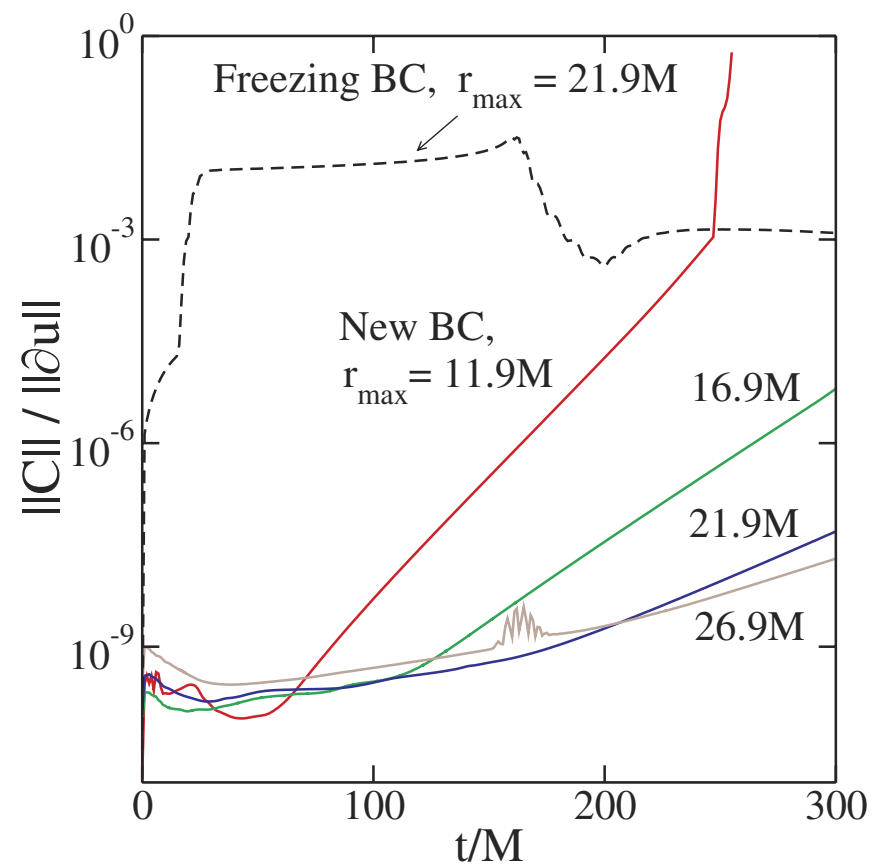

FIG. 9 (color online). Constraint violations in evolutions of a perturbed black hole. The dashed curve corresponds to freezing boundary conditions, and the solid curves correspond to the new boundary conditions for different $r_{\max }$.

The second interesting feature seen in Figs. 7 and 8 is the complicated structure of the curves starting at about $t \approx$ $20 M$, when the gravitational wave passes through the outer boundary. This structure seems to be caused by the (very weak) reflection of the gravitational-wave pulse back into very short-wavelength ingoing waves. Consider an outgoing wave with coordinate velocity near one (the speed of light) as it approaches the outer boundary. Since the nonlinear evolution equations and our boundary conditions couple the various characteristic fields, this wave will be mixed with and partially reflected as an ingoing wave which propagates at the much smaller shift speed $N^{i} n_{i} \ll$ 1 , even in the continuum limit. If the original outgoing wave has wavelength $\lambda$, then the reflected incoming wave that propagates at the shift speed will have the much smaller wavelength $\lambda N^{i} n_{i} \ll \lambda$. As the outer boundary radius is increased, the amplitude of these reflected waves is decreased. But their wavelength also decreases (because the shift $N^{i}$ decreases as $r$ increases) and therefore these reflected waves become more difficult to resolve numerically for larger $r_{\max }$.

One approach is to ignore these very small amplitude nonphysical reflected waves and not even attempt to resolve them. However, these reflected waves contribute (slightly) to the constraint quantities, so leaving them unresolved would introduce constraint violations that are roughly the size of the reflected waves. If this constraint violation is smaller than the numerical truncation error in the remainder of the domain, then no harm is done by ignoring the reflected waves. However, for the pseudospectral simulations presented here, the truncation error is so small elsewhere that the contributions of the reflected waves can dominate, obscuring our convergence tests. Therefore, we choose to resolve the reflected waves in the tests presented here.

Another approach would be to attempt to eliminate the problem completely by making a smarter choice for the shift $N^{i}$. The radial component of the shift could be made to approach a constant value rather than falling to zero as $r$ increases. This would limit the amount of blueshift the reflected waves could experience. Alternatively the radial component of the shift could be made to pass through zero and switch sign [18], thus eliminating the shift-speed incoming waves completely. These changes would either limit or entirely eliminate the problem, but possibly at the expense of introducing other gauge-related difficulties. Since the choice of gauge is not the main subject of this paper, we decided to deal with the reflected-wave problem here by increasing the resolution in the subdomains near the outer boundary. For instance, the $r_{\max }=26.9 \mathrm{M}$ curve in Fig. 9 was produced using eight subdomains with boundaries at $r=1.9 M, 6.9 M, 11.9 M, 16.9 M, 19.4 M$, $21.9 M, 23.567 M, 25.233 M$, and $26.9 M$, each using $N_{r}=$ 51 collocation points.

\section{Angular instability}

In addition to the constraint-violating instability and the gauge-mode instability discussed in Secs. VIII A and VIII B, there are also signs of a very weak instability that primarily affects the highest angular modes of the evolved fields. This angular instability has a growth rate that increases with increasing $\ell_{\max }$, and so this instability appears to be nonconvergent. This instability is not evident in any of the figures shown so far, and is negligible on the time scales of interest here except for simulations using very large $\ell_{\max }$ and very small $r_{\max }$. In order to see this instability clearly, we look at a quantity that is linear in the dynamical fields and vanishes unless the instability is present. To this end we define

$$
\delta \bar{K} \equiv \bar{g}^{i j}\left(K_{i j}-\bar{K}_{i j}\right),
$$

where $\bar{g}^{i j}$ and $\bar{K}_{i j}$ are exact solutions for the three-metric and extrinsic curvature, and $K_{i j}$ is the numerical extrinsic curvature from our simulations. To see the angular instability we project $\delta \bar{K}$ onto the spherical-harmonic basis at each radial collocation point $r_{p}$,

$$
\delta \bar{K}_{p \ell m}=\int Y_{\ell m}(\theta, \varphi) \delta \bar{K}\left(r_{p}, \theta, \varphi\right) \sin \theta d \theta d \varphi,
$$

and then average these over $r_{p}$ and $m$ by forming

$$
\left(\delta \bar{K}_{\ell}^{\mathrm{rms}}\right)^{2}=N_{r}^{-1}(2 \ell+1)^{-1} \sum_{p,|m| \leq \ell}\left(\delta \bar{K}_{p \ell m}\right)^{2} .
$$


Figure 10 shows the highest unfiltered components of $\delta \bar{K}_{\ell}^{\text {rms }}$ for evolutions of unperturbed black holes. These evolutions use our new boundary conditions, and are performed on a single computational subdomain with $N_{r}=$ 26 and $r_{\max }=6.9 M$. Each curve in Fig. 10 is generated from a run with a different $\ell_{\max }$, and the $\delta \bar{K}_{\ell}^{\mathrm{rms}}$ plotted is the one with $\ell=\ell_{\max }-4$, the largest $\ell$ that is untouched by our angular filtering procedure. Each $\ell$ component grows exponentially at a rate that increases with $\ell$. (The extremely rapid growth in the $\ell=10,12$, and 15 components that occurs just before the simulation crashes is presumably due to nonlinear coupling that becomes important only when the gauge mode becomes large.) For all cases plotted in Fig. 10 except $\ell_{\max }=21$, the simulation crashes because of the (convergent) gauge-mode instability described earlier. For these cases the angular instability is orders of magnitude smaller than the unstable gauge mode for the entire duration of the simulation. Only for $\ell_{\max }=$ 21 does the angular instability dominate before the end of the simulation.

We have explored the behavior of this angular instability in two ways. First we verified that the growth rate for a given $\ell$ component of $\delta \bar{K}_{\ell}^{\mathrm{rms}}$ is independent of the $\ell_{\max }$ used to compute it. We did this by comparing the curves in Fig. 10 with graphs of the same quantities computed from a single run with $\ell_{\max }=21$. The resulting plot looks almost identical to Fig. 10, except at very late times when the

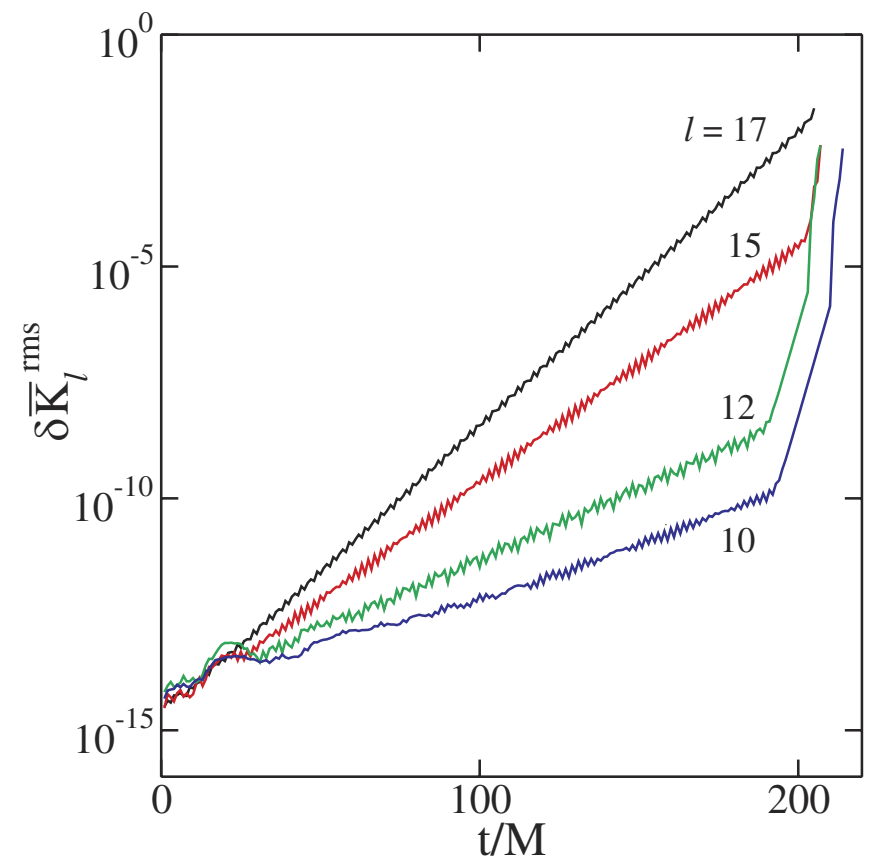

FIG. 10 (color online). Highest unfiltered components of $\delta \bar{K}_{\ell}^{\mathrm{rms}}$, defined in Eq. (101), for evolutions of unperturbed black holes. These evolutions use a single subdomain with $N_{r}=26$ and $r_{\max }=6.9 \mathrm{M}$. simulations begin to crash. And second, we explored the growth rates of the angular instability for fixed $\ell$ as a function of $r_{\max }$. Figure 11 shows $\delta \bar{K}_{17}^{\mathrm{rms}}$ for runs with different $r_{\max }$. We increased $r_{\max }$ in these runs by adding more subdomains of width $5 \mathrm{M}$, each having $N_{r}=26$ and $\ell_{\max }=21$. Because the angular instability is largest near the outer boundary, we compute $\delta \bar{K}_{17}^{\mathrm{rms}}$ only in the outermost subdomains for these plots.

The curves in Fig. 11 show that the angular instability becomes weaker and weaker as we increase the size of the computational domain. To study this behavior quantitatively, we plot the exponential growth rates of $\delta \bar{K}_{17}^{\mathrm{rms}}$ as a function of $r_{\max }$; these are shown as circles in Fig. 12. The error bars in the inset correspond to the variation in slopes that we extract from data segments of different length, but these error bars are not shown in the main plot in Fig. 12 because they are the same size as the plot symbols. Rather than falling off gradually like $1 / r_{\max }$ as might have been expected, the growth rate in Fig. 12 appears to go to zero at a finite value of $r_{\max }$. This finite value of $r_{\max }$ depends on $\ell$; to show this dependence, we also plot in Fig. 12 the exponential growth rates of $\delta \bar{K}_{12}^{\mathrm{rms}}$ (shown as triangles in the plot) for the same runs. The best linear fits through the $\ell=17$ and $\ell=12$ growth rates have the same slope, but the growth rate of the $\ell=12$ mode goes to zero at a smaller $r_{\max }$. Figure 12 suggests that for a given $\ell_{\max }$ there are no angular instabilities at all when $r_{\max }$ is sufficiently

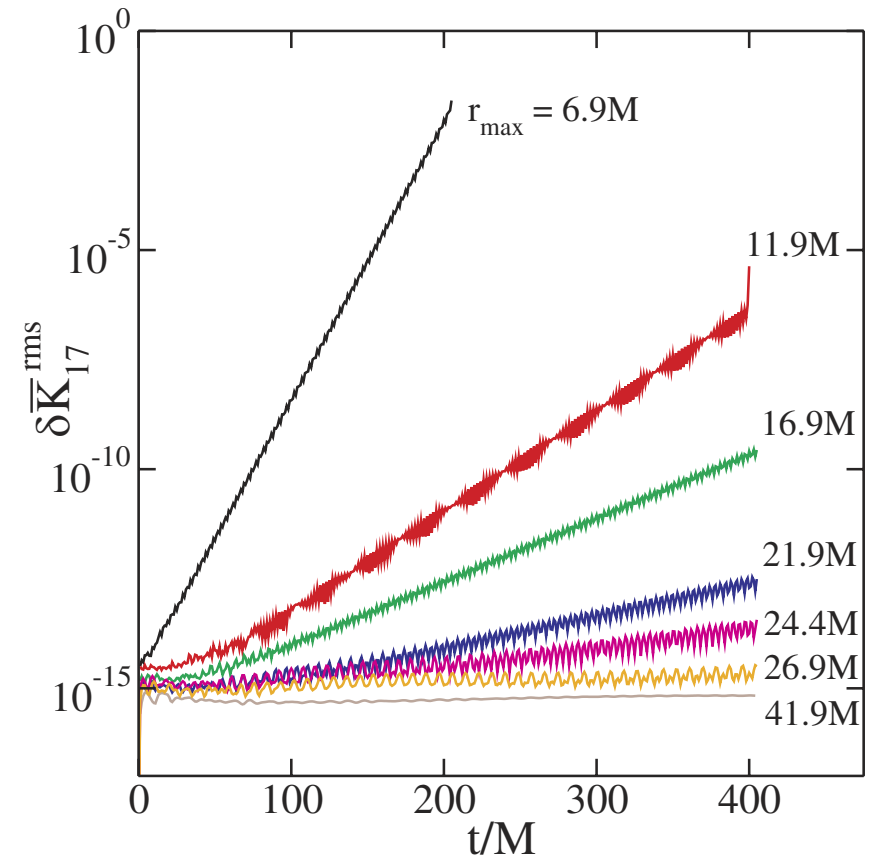

FIG. 11 (color online). $\quad \delta \bar{K}_{17}^{\text {rms }}$ for unperturbed black-hole evolutions on domains with different $r_{\max }$. This quantity is evaluated on the outermost domain which has width $5 M, N_{r}=26$, and $\ell_{\max }=21$. These evolutions use our new boundary conditions. 


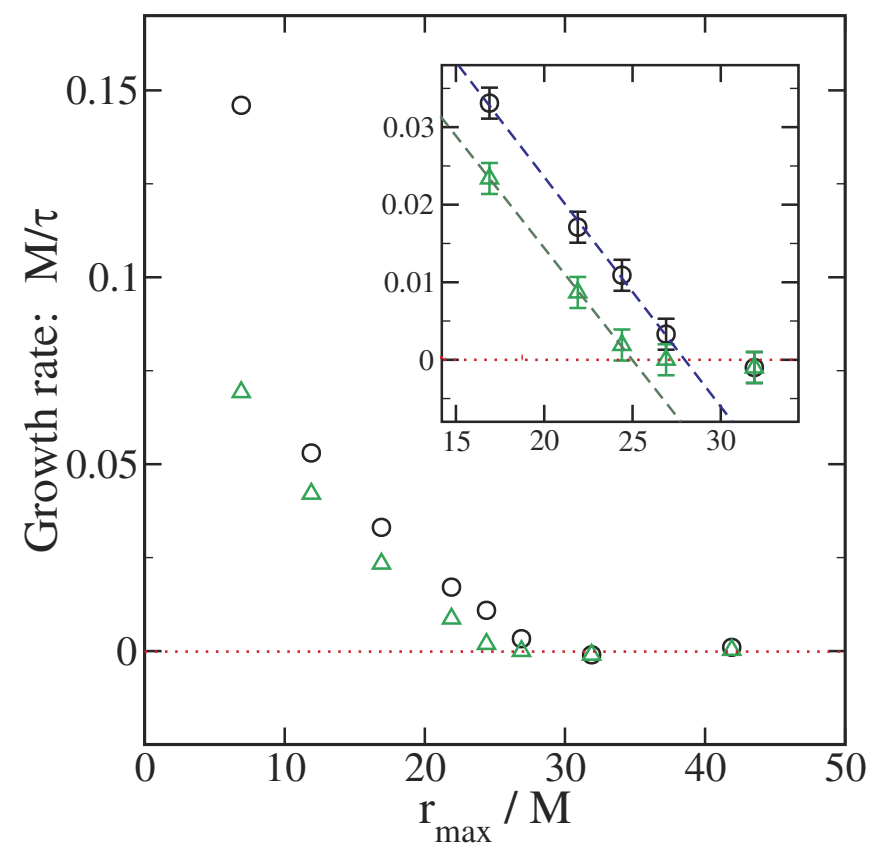

FIG. 12 (color online). Exponential growth rate of $\delta \bar{K}_{17}^{\mathrm{rms}}$ (circles) and $\delta \bar{K}_{12}^{\mathrm{rms}}$ (triangles) for different $r_{\max }$ in evolutions of unperturbed black holes with our new boundary conditions. The inset shows an enlargement with the best linear fits through the points $16.9 M \leq r_{\max } \leq 26.9 M$.

large. No angular instability has been detected in any of our runs with $\ell_{\max } \leq 21, t \leq 300 M$, and $r_{\max }>27 M$. Our results also suggest that for a given $r_{\max }$, an angular instability with arbitrarily large growth rate could be found by making $\ell_{\max }$ sufficiently large.

We see no angular instability at all when we use freezing boundary conditions. Furthermore, the angular instability shown in Figs. 10-12 is present whether or not we use the physical boundary condition, Eq. (78), or the gauge boundary conditions, Eqs. (80) and (82). [Although as noted before, an angular instability dominates when Eq. (82) is imposed on all the spherical-harmonic components of $U^{1-}$.] It is unclear whether this angular instability is due to our numerical method or whether the new constraintpreserving boundary conditions yield an ill-posed initialboundary-value problem at the continuum level. We note however that for the resolutions and time scales of interest here, this instability remains small and can be controlled quite effectively by modestly increasing the radius of the outer boundary. And the numerical evolutions, such as those in Figs. 7-9, produced by these methods do appear to be accurate solutions of the Einstein equations: both the constraints $\|C\| /\|\partial u\|$ and the four-dimensional Ricci tensor $\|R\| /\left\|R^{\mathrm{rms}}\right\|$ can be made arbitrarily small. Therefore from a practical point of view it may not matter whether these boundary conditions are formally well posed, or that our computational methods contain a very mild nonconvergent angular instability.

\section{DISCUSSION}

This paper constructs new boundary conditions for the KST form of the Einstein evolution system that are designed to prevent the influx of constraint violations and physical gravitational waves into the computational domain. From a mathematical point of view, these boundary conditions are Neumann like (in the sense that they place conditions on the normal derivatives of the incoming characteristic fields). Boundary conditions of this type have not been studied as comprehensively as the simpler "maximally dissipative" boundary conditions (which are Dirichlet like in that they place conditions on the values of incoming characteristic fields themselves). Rigorous mathematical well-posedness theorems do not yet exist for hyperbolic evolution systems with these Neumannlike boundary conditions. So additional mathematical analysis of these conditions is urgently needed to determine whether they are well posed both at the continuum differential equation level and the discrete numerical level. Should this analysis reveal that these conditions are ill posed, then alternate ways of preventing the influx of constraint violations and physical gravitational waves in these systems would be needed even more urgently.

Our numerical tests of these new constraint-preserving and physical boundary conditions show them to be quite effective: constraint violations can be reduced to roundofflevel errors in dynamical black-hole evolutions. Nevertheless, several weak instabilities did appear in our numerical results, and additional work is needed to sort out exactly what their causes are and what methods can be used to control them. Are the constraint-violating instabilities seen in the evolutions of Figs. 3 and 9 really caused by bulk constraint-violating terms in the constraint evolution equations (as we surmise), and can optimal constraint projection methods such as those developed for the scalar field system [3] be used to control them? Can the gauge instabilities seen in Fig. 6 be controlled by the introduction of dynamical evolution equations for the lapse and shift? Are the nonconvergent angular instabilities seen in Figs. 10 and 11 a symptom of ill posedness of these boundary conditions at the continuum or the discrete numerical level? And what can be done to cure these problems?

\section{ACKNOWLEDGMENTS}

We thank Olivier Sarbach, Saul Teukolsky, and Manuel Tiglio for helpful discussions concerning this work. Some of the computations for this project were performed with the Tungsten cluster at NCSA. This work was supported in part by NSF Grants No. PHY-0099568, No. PHY-0244906 and NASA Grants No. NAG5-10707, No. NAG5-12834 at Caltech, and NSF Grants No. PHY-0312072, No. PHY0354631 at Cornell. L. T. B. was supported by the National Research Council at the Jet Propulsion Laboratory, California Institute of Technology. 


\section{APPENDIX A: KST CHARACTERISTIC FIELDS}

Explicit expressions are given here for the characteristic fields of the KST form of the Einstein evolution system. These characteristic fields consist of the collection $u^{\hat{\alpha}}=$ $\left\{g_{i j}, Z^{1}, Z_{i}^{2}, Z_{i}^{3}, Z_{i}^{4}, Z_{i j}^{5}, Z_{k i j}^{6}, U^{1 \pm}, U_{i}^{2 \pm}, U^{3 \pm}, U_{i j}^{4 \pm}\right\}$, and can be written in terms of the principal evolution fields, Eq. (7):

$$
\begin{aligned}
& Z^{1}=\gamma_{3} n^{i} D_{i}^{1}-2\left(1+\gamma_{4}\right) n^{i} D_{i}^{2}, \\
& Z_{i}^{2}=\gamma_{4} P^{j}{ }_{i} D_{j}^{1}-\left(\gamma_{3}+2 \gamma_{4}\right) P^{j}{ }_{i} n^{k} n^{l} D_{j k l}, \\
& Z_{i}^{3}=3 P^{j}{ }_{i} D_{j}^{1}-2 P^{j}{ }_{i} D_{j}^{2}-4 P^{j}{ }_{i} n^{k} n^{l} D_{j k}, \\
& Z_{i}^{4}=48 v_{2}^{2} n^{l} P_{i}^{j} n^{k} D_{l j k}+2 \gamma_{4}\left(5-9 \gamma_{2}\right) P^{j}{ }_{i} D_{j}^{2} \\
& +3\left(1-3 \gamma_{2}-4 \gamma_{0}\right)\left(4-\gamma_{3}\right)\left(P^{j}{ }_{i} D_{j}^{1}+P^{j}{ }_{i} n^{k} n^{l} D_{j k l}\right) \\
& -2\left(6+\gamma_{4}\right)\left(5-9 \gamma_{2}\right) P_{i}^{j} n^{k} n^{l} D_{j k l} \text {, } \\
& Z_{i j}^{5}=\left(P_{i}^{a} P_{j}^{b}-\frac{1}{2} P_{i j} P^{a b}\right) n^{k} D_{a b k}, \\
& Z_{k i j}^{6}=P_{k i j}^{c a b} D_{c a b} \\
& U^{1 \pm}= \pm\left[1+2 v_{1}^{2}+\left(1+2 \gamma_{1}\right) q\right] n^{i} D_{i}^{1} \\
& -v_{1}(1-q) P^{i j} K_{i j}+2 v_{1} n^{i} n^{j}\left[K_{i j} \pm v_{1} n^{k} D_{k i j}\right] \\
& \mp\left[1-3 \gamma_{2}+\left(1+2 \gamma_{1}+\gamma_{2}\right) q\right] n^{i} D_{i}^{2} \text {, } \\
& U_{i}^{2 \pm}= \pm 2 v_{2} n^{k} P^{j}{ }_{i} K_{j k}+\left(1+2 \gamma_{0}\right) P^{j}{ }_{i} D_{j}^{1} \text {, } \\
& -\left(1-\gamma_{2}\right) P_{i}^{j} D_{j}^{2}+\left(2 \gamma_{0}-\gamma_{2}\right) P^{j}{ }_{i} n^{k} n^{l} D_{j k l} \text {, } \\
& U^{3 \pm}= \pm\left(1+2 \gamma_{1}\right) n^{i} D_{i}^{1} \mp\left(1+2 \gamma_{1}+\gamma_{2}\right) n^{i} D_{i}^{2} \\
& +v_{3} P^{i j} K_{i j} \text {, } \\
& U_{i j}^{4 \pm}=\left(P^{a}{ }_{i} P_{j}^{b}-\frac{1}{2} P_{i j} P^{a b}\right) \\
& \times\left[K_{a b} \pm n^{k} D_{k a b} \mp\left(1+\gamma_{2}\right) n^{k} D_{(a b) k}\right] .
\end{aligned}
$$

In these expressions the two distinct traces of $D_{k i j}$ are written as

$$
\begin{aligned}
& D_{i}^{1}=P^{j k} D_{i j k}, \\
& D_{i}^{2}=P^{j k} D_{k i j},
\end{aligned}
$$

where

$$
P^{i j}=g^{i j}-n^{i} n^{j},
$$

is the projection orthogonal to $n_{i}$. The quantities $v_{1}, v_{2}$ and $v_{3}$ are defined in Eqs. (8)-(10), and $q$ is given by

$$
q=\frac{1+3 v_{1}^{2}-4 v_{2}^{2}}{v_{1}^{2}-v_{3}^{2}}
$$

Finally, the projection tensor $P_{k i j}^{c a b}$ is defined by

$$
\begin{aligned}
P_{k i j}^{c a b}= & P_{k}^{c} P_{i}^{(a} P_{j}^{b)}-\frac{3}{4} P_{i j} P_{k}^{c} P^{a b}+\frac{1}{2} P^{c(a} P_{k}^{b)} P_{i j} \\
& +\frac{1}{2} P^{a b} P_{(i}^{c} P_{j) k}-P^{c(a} P^{b)}{ }_{(i} P_{j) k} .
\end{aligned}
$$

These expressions are the completely general forms of the characteristic fields for the KST system. They reduce to the expressions for the restricted case $v_{1}^{2}=v_{2}^{2}=v_{3}^{3}=1$ published previously in Ref. [29].

The characteristic fields $U^{1 \pm}, U_{i}^{2 \pm}$, and $U^{3 \pm}$ have characteristic speeds $\pm \boldsymbol{v}_{1}, \pm \boldsymbol{v}_{2}$, and $\pm \boldsymbol{v}_{3}$ respectively (relative to the hypersurface normal observers); the fields $U_{i j}^{4 \pm}$ have speeds \pm 1 ; the fields $\left\{Z^{1}, Z_{i}^{2}, Z_{i}^{3}, Z_{i}^{4}, Z_{i j}^{5}, Z_{k i j}^{6}\right\}$ all have characteristic speed zero. The characteristic fields are linearly independent (so the KST evolution system is strongly hyperbolic) if $v_{1} \neq 0, v_{2} \neq 0, v_{3} \neq 0$, and $v_{1} \neq v_{3}$. In the case when $v_{1}=v_{3}$, the characteristic fields $U^{1 \pm}$ given in Eq. (A7) are not defined because the quantity $q$ given in Eq. (A14) is not defined. We find that there are nevertheless a complete set of characteristic fields in the $v_{1}=v_{3} \neq 0$ case so long as

$$
1+3 v_{1}^{2}=4 v_{2}^{2}
$$

In this case, the characteristic fields $U^{1 \pm}$ are just given by the expression in Eq. (A7) with $q=0$. Any other constant value of $q$ is also acceptable, with the result being a redefinition of $U^{1 \pm}$ by the addition of $q$ times $U^{3 \pm}$ (which has the same characteristic speed in this case). The choice $q=0$ is probably the simplest, but other choices might be desirable under some circumstances. For example, for symmetric hyperbolic systems it might be better to make the eigenvectors mutually orthogonal in terms of the symmetrizer metric. The symmetric hyperbolicity of the KST system is discussed in Appendix B of Ref. [32].

It is also useful to have explicit expressions for the inverse transformation, $u^{\alpha}=e^{\alpha}{ }_{\hat{\beta}} u^{\hat{\beta}}$, that expresses the principal evolution fields in terms of the characteristic fields. These inverse transformations for the general KST form of the Einstein evolution system are given by

$$
\begin{aligned}
K_{i j}= & n_{i} n_{j}\left[\frac{1-q}{4 v_{3}}\left(U^{3+}+U^{3-}\right)+\frac{U^{1+}+U^{1-}}{4 v_{1}}\right] \\
& +\frac{n_{(i} U_{j)}^{2+}-n_{(i} U_{j)}^{2-}}{2 v_{2}}+P_{i j} \frac{U^{3+}+U^{3-}}{4 v_{3}} \\
& +\frac{1}{2}\left(U_{i j}^{4+}+U_{i j}^{4-}\right),
\end{aligned}
$$




$$
\begin{aligned}
D_{k i j}= & n_{k} n_{i} n_{j} n^{c} n^{a} n^{b} D_{c a b}+n_{i} n_{j} P^{c}{ }_{k} n^{a} n^{b} D_{c a b} \\
& +2 n_{k} n_{(i} P^{a}{ }_{j)} n^{b} n^{c} D_{c a b}+\frac{1}{2} n_{k}\left(U_{i j}^{4+}-U_{i j}^{4-}\right) \\
& +\left(1+\gamma_{2}\right) n_{k} Z_{(i j)}^{5}+2 Z_{k(i}^{5} n_{j)}+Z_{k i j}^{6} \\
& +\frac{1}{2} n_{k} P_{i j} n^{c} D_{c}^{1}+\frac{3}{4} P_{i j} P_{k}^{c} D_{c}^{1}-\frac{1}{2} P_{k(i} P_{j)}^{c} D_{c}^{1} \\
& +n_{(i} P_{j) k} n^{c} D_{c}^{2}-\frac{1}{2} P_{i j} P_{k}^{c} D_{c}^{2}+P_{k(i} P_{j)}^{c} D_{c}^{2} .
\end{aligned}
$$

The terms involving $D_{k i j}$ on the right sides of Eq. (A18) are given by

$$
\begin{aligned}
n^{c} n^{a} n^{b} D_{c a b}= & {\left[v_{1}^{2}\left(1+2 \gamma_{1}+\gamma_{2}\right)+\gamma_{2}\left(2+3 \gamma_{1}\right)\right] \frac{Z^{1}}{2 v_{1}^{2} v_{3}^{2}} } \\
& +\frac{1+\gamma_{3}+\gamma_{4}-q}{4 v_{3}^{2}}\left(U^{3+}-U^{3-}\right) \\
& +\frac{U^{1+}-U^{1-}}{4 v_{1}^{2}},
\end{aligned}
$$

$$
\begin{aligned}
P^{c}{ }_{i} n^{a} n^{b} D_{c a b}= & -\frac{1-3 \gamma_{2}-4 \gamma_{0}}{8 v_{2}^{2}} Z_{i}^{2}+\frac{\gamma_{4}\left(1-\gamma_{2}\right)}{8 v_{2}^{2}} Z_{i}^{3} \\
& -\frac{\gamma_{4}}{8 v_{2}^{2}}\left(U_{i}^{2+}+U_{i}^{2-}\right),
\end{aligned}
$$$$
P^{a}{ }_{i} n^{c} n^{b} D_{c a b}=-\frac{5-9 \gamma_{2}}{16 v_{2}^{2}} Z_{i}^{2}+\frac{4-\gamma_{3}}{16 v_{2}^{2}}
$$$$
\times\left(U_{i}^{2+}+U_{i}^{2-}\right)-\left[3\left(1-\gamma_{2}\right)\left(4-\gamma_{3}\right)\right.
$$$$
\left.-\gamma_{4}\left(5-9 \gamma_{2}\right)\right] \frac{Z_{i}^{3}}{48 v_{2}^{2}}+\frac{Z_{i}^{4}}{48 v_{2}^{2}} \text {, }
$$$$
n^{c} D_{c}^{1}=\frac{1+\gamma_{4}}{2 v_{3}^{2}}\left(U^{3+}-U^{3-}\right)-\left(1+2 \gamma_{1}+\gamma_{2}\right) \frac{Z^{1}}{2 v_{3}^{2}},
$$$$
n^{c} D_{c}^{2}=\frac{\gamma_{3}}{4 v_{3}^{2}}\left(U^{3+}-U^{3-}\right)-\left(1+2 \gamma_{1}\right) \frac{Z^{1}}{2 v_{3}^{2}},
$$

$$
\begin{aligned}
P_{i}^{c} D_{c}^{1}= & \left(1-\gamma_{2}\right)\left(\gamma_{3}+2 \gamma_{4}\right) \frac{Z_{i}^{3}}{8 v_{2}^{2}}-\left(2-3 \gamma_{2}+2 \gamma_{0}\right) \frac{Z_{i}^{2}}{4 v_{2}^{2}} \\
& -\frac{\gamma_{3}+2 \gamma_{4}}{8 v_{2}^{2}}\left(U_{i}^{2+}+U_{i}^{2-}\right), \\
P_{i}^{c} D_{c}^{2}= & {\left[\gamma_{3}\left(1+2 \gamma_{0}\right)+\gamma_{4}\left(2-\gamma_{2}+6 \gamma_{0}\right)\right] \frac{Z_{i}^{3}}{8 v_{2}^{2}} } \\
& -\left(3 \gamma_{3}+2 \gamma_{4}\right) \frac{U_{i}^{2+}+U_{i}^{2-}}{16 v_{2}^{2}} \\
& -\left(4-3 \gamma_{2}+14 \gamma_{0}\right) \frac{Z_{i}^{2}}{8 v_{2}^{2}} .
\end{aligned}
$$

\section{APPENDIX B: HYPERBOLICITY OF CONSTRAINT EVOLUTION}

In this Appendix we evaluate the hyperbolicity of the KST constraint evolution system, Eqs. (15)-(18). This system can be written in the more abstract form

$$
\partial_{t} c^{A}+A_{B}^{k A} \partial_{k} c^{B}=F_{B}^{A} c^{B} .
$$

In Sec. III we demonstrated the strong hyperbolicity of the KST constraint evolution system by showing that the characteristic matrices $n_{k} A^{k A}{ }_{B}$ have a complete set of eigenvectors. Here we determine the conditions under which this constraint evolution system is also symmetric hyperbolic, i.e., that there exists a symmetric positive definite $S_{A B}$ (the symmetrizer) with the property that it symmetrizes the characteristic matrices: $A_{A B}^{k} \equiv S_{A C} A_{B}^{k C}=A_{B A}^{k}$.

The most general symmetrizer for the constraint evolution system can be written conveniently by defining the following quantities associated with the constraint $C_{k l i j}$ :

$$
\mathcal{D}_{k i j}=\epsilon_{k}{ }^{a b} C_{a b i j},
$$

where $\epsilon_{k i j}$ is the spatial volume element. We also define

$$
\begin{gathered}
\mathcal{D}_{i}^{1}=g^{j k} \mathcal{D}_{i j k}, \\
\mathcal{D}_{i}^{2}=g^{j k} \mathcal{D}_{j i k}, \\
\tilde{\mathcal{D}}_{k i j}=\mathcal{D}_{k i j}+\frac{1}{5}\left[\mathcal{D}_{(i}^{1} g_{j) k}-2 \mathcal{D}_{k}^{1} g_{i j}+\mathcal{D}_{k}^{2} g_{i j}\right. \\
\left.-3 \mathcal{D}_{(i}^{2} g_{j) k}\right] .
\end{gathered}
$$

Then the most general symmetrizer constructed from the metric $g_{i j}$ is

$$
\begin{aligned}
d S^{2}= & S_{A B} d c^{A} d c^{B} \\
= & \chi_{1} d C^{2}+\chi_{2} g^{i a} d C_{i} d C_{a}+\chi_{3} g^{i a} g^{j b} g^{k c} d \tilde{\mathcal{D}}_{(k i j)} d \tilde{\mathcal{D}}_{(c a b)}+\chi_{4} g^{i a} g^{j b} g^{k c}\left[d \tilde{\mathcal{D}}_{k i j}-d \tilde{\mathcal{D}}_{(k i j)}\right]\left[d \tilde{\mathcal{D}}_{c a b}-d \tilde{\mathcal{D}}_{(c a b)}\right] \\
& +\chi_{5} g^{i a} d \mathcal{D}_{i}^{1} d \mathcal{D}_{a}^{1}+\chi_{6} g^{i a} d \mathcal{D}_{i}^{2} d \mathcal{D}_{a}^{2}+2 \chi_{7} g^{i a} d \mathcal{D}_{i}^{1} d \mathcal{D}_{a}^{2} .
\end{aligned}
$$


The conditions needed to ensure that this symmetrizer is positive definite are $\chi_{a}>0$ for $a=1, \ldots, 6$ and $\chi_{5} \chi_{6}>$ $\chi_{7}^{2}$. There are no cross terms between the momentum constraint $C_{i}$ and the $\mathcal{D}_{i}^{1}$ or $\mathcal{D}_{i}^{2}$ constraints, because the resulting terms would have the wrong parity. Using the principal parts of the KST constraint evolution system given in Eqs. (15)-(18), we find that the conditions needed for symmetric hyperbolicity are

$$
\begin{gathered}
0=\chi_{1}\left(1-\frac{1}{2} \gamma_{3}+\gamma_{4}\right)-\chi_{2}\left(1+2 \gamma_{1}\right), \\
0=2 \chi_{5}\left(\gamma_{3}+3 \gamma_{4}\right)+\chi_{2} \gamma_{0}+\chi_{7}\left(2 \gamma_{4}-\gamma_{3}\right), \\
0=\frac{1}{2} \chi_{2}-\chi_{6}\left(\gamma_{3}-2 \gamma_{4}\right)+\chi_{7}\left(2 \gamma_{3}+6 \gamma_{4}\right), \\
0=\frac{1}{2} \chi_{2} \gamma_{2}+\chi_{4} \gamma_{3} .
\end{gathered}
$$

The problem now is to determine when these symmetrization conditions can be satisfied. For simplicity we focus attention here on the case where all of the characteristic speeds of the principal evolution system have the physical values: $0, \pm 1$. These conditions on the characteristic speeds imply the following conditions on the parameters:

$$
\begin{gathered}
\gamma_{0}=\frac{1}{2}, \\
\gamma_{3}=\frac{-8}{4 \gamma_{2}+\left(5+3 \gamma_{2}\right)\left(1+2 \gamma_{1}\right)}, \\
\gamma_{4}=\frac{1-\gamma_{2}-\left(1+2 \gamma_{1}\right)\left(5+3 \gamma_{2}\right)}{4 \gamma_{2}+\left(5+3 \gamma_{2}\right)\left(1+2 \gamma_{1}\right)} .
\end{gathered}
$$

The analysis in Lindblom and Scheel [41] shows that the principal evolution system is symmetric hyperbolic in this case for all values of the parameters $\gamma_{1}$ and $\gamma_{2}$ that satisfy the following inequalities:

$$
\begin{gathered}
-\frac{5}{3}<\gamma_{2}<0, \\
4 \gamma_{2}+\left(5+3 \gamma_{2}\right)\left(1+2 \gamma_{1}\right) \neq 0 .
\end{gathered}
$$

Substituting the conditions Eqs. (B11)-(B13) into the symmetry conditions, Eqs. (B7)-(B10), we find the following conditions on the $\chi_{a}$ :

$$
\begin{gathered}
\chi_{2}=\frac{\chi_{1}\left(5+3 \gamma_{2}\right)}{\left(1+2 \gamma_{1}\right)\left[4 \gamma_{2}+\left(5+3 \gamma_{2}\right)\left(1+2 \gamma_{1}\right)\right]}, \\
\chi_{4}=\frac{\chi_{1} \gamma_{2}\left(5+3 \gamma_{2}\right)}{16\left(1+2 \gamma_{1}\right)},
\end{gathered}
$$

$$
\begin{gathered}
\chi_{5}=\frac{\chi_{1}\left(5+3 \gamma_{2}\right)-8 \chi_{7}\left(1+2 \gamma_{1}\right)\left[2 \gamma_{2}+\gamma_{1}\left(5+3 \gamma_{2}\right)\right]}{8\left(5+3 \gamma_{2}\right)\left(2+7 \gamma_{1}+6 \gamma_{1}^{2}\right)} \\
\chi_{6}=\frac{\left(5+3 \gamma_{2}\right)\left[\chi_{1}-8 \chi_{7}\left(2+7 \gamma_{1}+6 \gamma_{1}^{2}\right)\right]}{8\left(1+2 \gamma_{1}\right)\left[2 \gamma_{2}+\gamma_{1}\left(5+3 \gamma_{2}\right)\right]} .
\end{gathered}
$$

The constraint evolution system is symmetric hyperbolic if and only if $\chi_{a}>0$ for $a=\{1,2,3,4,5,6\}$ and $\chi_{5} \chi_{6}>\chi_{7}^{2}$. If the principal evolution system is symmetric hyperbolic then $5+3 \gamma_{2}>0$ [from Eq. (B14)], thus we see from Eq. (B17) that we must have

$$
1+2 \gamma_{1}<0,
$$

or equivalently $\gamma_{1}<-\frac{1}{2}$ if the constraint evolution system is to be symmetric hyperbolic as well. This condition guarantees that $4 \gamma_{2}+\left(5+3 \gamma_{2}\right)\left(1+2 \gamma_{1}\right)<0$ and so the second inequality in Eq. (B15) is automatically satisfied in this case. Consequently Eq. (B16) places no further restrictions on the parameters $\gamma_{1}$ and $\gamma_{2}$.

To analyze the inequalities on $\chi_{5}$ and $\chi_{6}$ implied by Eqs. (B18) and (B19), we restrict our attention to the simple case where $\chi_{7}$ can be set to zero. In this case Eqs. (B18) and (B19) simplify considerably:

$$
\begin{gathered}
\chi_{5}=\frac{\chi_{1}}{8\left(2 \gamma_{1}+1\right)\left(3 \gamma_{1}+2\right)}, \\
\chi_{6}=\frac{\left(5+3 \gamma_{2}\right) \chi_{1}}{8\left(1+2 \gamma_{1}\right)\left[2 \gamma_{2}+\gamma_{1}\left(5+3 \gamma_{2}\right)\right]} .
\end{gathered}
$$

Equation (B22) guarantees that the coefficient $\chi_{6}$ is positive without any additional restrictions, while Eq. (B21) shows that the parameter $\gamma_{1}$ must be further restricted by the inequality

$$
\gamma_{1}<-\frac{2}{3},
$$

in order to ensure that $\chi_{5}$ is positive. This argument shows then that we can choose the parameters $\chi_{a}>0$ for $a=$ $\{1,2,3,4,5,6\}$ and $\chi_{7}=0$ for any values of the parameters $\gamma_{1}$ and $\gamma_{2}$ in the ranges:

$$
\begin{gathered}
\gamma_{1}<-\frac{2}{3}, \\
-\frac{5}{3}<\gamma_{2}<0 .
\end{gathered}
$$

We have not yet found the minimal set of restrictions on the parameters that allows the KST constraint evolution system to be symmetric hyperbolic for any values of the characteristic speeds, and for any values of the parameter $\chi_{7}$. We have limited our numerical experiments so far to the regions of this parameter space where both the principal and the constraint evolution systems are known to be symmetric hyperbolic. 
[1] E. Schnetter, Ph.D. thesis, Universität Tübingen, 2003, grqc/0411002.

[2] M. Anderson and R. A. Matzner, gr-qc/0307055.

[3] M. Holst, L. Lindblom, R. Owen, H. P. Pfeiffer, M. A. Scheel, and L. E. Kidder, Phys. Rev. D 70, 084017 (2004).

[4] T. Piran and R.F. Stark, in Dynamical Spacetimes and Numerical Relativity, edited by J.M. Centrella (Cambridge University Press, Cambridge, UK, 1986), pp. $40-73$.

[5] A. M. Abrahams and C. R. Evans, Phys. Rev. D 46, R4117 (1992).

[6] M. W. Choptuik, Phys. Rev. Lett. 70, 9 (1993).

[7] A. M. Abrahams and C. R. Evans, Phys. Rev. Lett. 70, 2980 (1993).

[8] A. M. Abrahams, G. B. Cook, S. L. Shapiro, and S. A. Teukolsky, Phys. Rev. D 49, 5153 (1994).

[9] M. W. Choptuik, E. W. Hirschmann, S. L. Liebling, and F. Pretorius, Classical Quantum Gravity 20, 1857 (2003).

[10] M. W. Choptuik, E. W. Hirschmann, S. L. Liebling, and F. Pretorius, Phys. Rev. D 68, 044007 (2003).

[11] S. Bonazzola, E. Gourgoulhon, P. Grandclement, and J. Novak, Phys. Rev. D 70, 104007 (2004).

[12] M. Tiglio, gr-qc/0304062.

[13] M. Tiglio, L. Lehner, and D. Neilsen, Phys. Rev. D 70, 104018 (2004).

[14] L. Lindblom, M. A. Scheel, L. E. Kidder, H. P. Pfeiffer, D. Shoemaker, and S. A. Teukolsky, Phys. Rev. D 69, 124025 (2004).

[15] H. Friedrich and G. Nagy, Commun. Math. Phys. 201, 619 (1999).

[16] M. S. Iriondo and O. A. Reula, Phys. Rev. D 65, 044024 (2002).

[17] J. M. Stewart, Classical Quantum Gravity 15, 2865 (1998).

[18] J. M. Bardeen and L.T. Buchman, Phys. Rev. D 65, 064037 (2002).

[19] B. Szilágyi, B. Schmidt, and J. Winicour, Phys. Rev. D 65 , 064015 (2002).

[20] G. Calabrese, L. Lehner, and M. Tiglio, Phys. Rev. D 65, 104031 (2002).

[21] B. Szilágyi and J. Winicour, Phys. Rev. D 68, 041501(R) (2003).

[22] G. Calabrese, J. Pullin, O. Sarbach, M. Tiglio, and O. Reula, Commun. Math. Phys. 240, 377 (2003).
[23] S. Frittelli and R. Gomez, Classical Quantum Gravity 20, 2379 (2003).

[24] S. Frittelli and R. Gomez, Phys. Rev. D 68, 044014 (2003).

[25] G. Calabrese and O. Sarbach, J. Math. Phys. (N.Y.) 44, 3888 (2003).

[26] S. Frittelli and R. Gomez, Phys. Rev. D 69, 124020 (2004).

[27] A. M. Alekseenko, gr-qc/0405080.

[28] S. Frittelli and O. A. Reula, Phys. Rev. Lett. 76, 4667 (1996).

[29] L.E. Kidder, M. A. Scheel, and S. A. Teukolsky, Phys. Rev. D 64, 064017 (2001).

[30] A. Anderson and J. W. York, Jr., Phys. Rev. Lett. 82, 4384 (1999).

[31] S. D. Hern, Ph.D. thesis, University of Cambridge, 1999, gr-qc/0004036.

[32] L. Lindblom and M. A. Scheel, Phys. Rev. D 67, 124005 (2003).

[33] M. Bjørhus, SIAM J. Sci. Comput. 16, 542 (1995).

[34] O. Reula and O. Sarbach, gr-qc/0409027.

[35] H. Friedrich, Classical Quantum Gravity 13, 1451 (1996).

[36] E. T. Newman and R. Penrose, J. Math. Phys. (N.Y.) 3, 566 (1962); 4, 998(E) (1963).

[37] M. Alcubierre and B. Brügmann, Phys. Rev. D 63, 104006 (2001).

[38] M. Alcubierre, B. Brügmann, P. Diener, M. Koppitz, D. Pollney, E. Seidel, and R. Takahashi, Phys. Rev. D 67, 084023 (2003).

[39] M. A. Scheel, A. L. Erickcek, L. M. Burko, L. E. Kidder, H.P. Pfeiffer, and S.A. Teukolsky, Phys. Rev. D 69, 104006 (2004).

[40] L. E. Kidder, M. A. Scheel, S. A. Teukolsky, E. D. Carlson, and G. B. Cook, Phys. Rev. D 62, 084032 (2000).

[41] L. Lindblom and M. A. Scheel, Phys. Rev. D 66, 084014 (2002).

[42] M. A. Scheel, L. E. Kidder, L. Lindblom, H. P. Pfeiffer, and S. A. Teukolsky, Phys. Rev. D 66, 124005 (2002).

[43] M.W. Choptuik, in Deterministic Chaos in General Relativity, edited by D. Hobell, A. Burd, and A. Coley (Plenum, New York, 1994), pp. 155-175.

[44] S. A. Teukolsky, Phys. Rev. D 26, 745 (1982).

[45] H. P. Pfeiffer, L.E. Kidder, M. A. Scheel, and D. Shoemaker, Phys. Rev. D 71, 024020 (2005). 